ARTICLE

https://doi.org/10.1038/s41467-020-17311-4

\title{
Nuclear moonlighting of cytosolic glyceraldehyde- 3-phosphate dehydrogenase regulates Arabidopsis response to heat stress
}

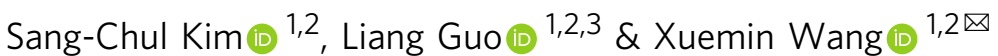

\begin{abstract}
Various stress conditions induce the nuclear translocation of cytosolic glyceraldehyde-3phosphate dehydrogenase (GAPC), but its nuclear function in plant stress responses remains elusive. Here we show that GAPC interacts with a transcription factor to promote the expression of heat-inducible genes and heat tolerance in Arabidopsis. GAPC accumulates in the nucleus under heat stress. Overexpression of GAPC enhances heat tolerance of seedlings and the expression of heat-inducible genes whereas knockout of GAPCs has opposite effects. Screening of Arabidopsis transcription factors identifies nuclear factor $Y$ subunit $\mathrm{C10}$ (NF$Y(10)$ as a GAPC-binding protein. The effects of GAPC overexpression are abolished when NF-YC10 is deficient, the heat-induced nuclear accumulation of GAPC is suppressed, or the GAPC-NF-YC10 interaction is disrupted. GAPC overexpression also enhances the binding ability of NF-YC10 to its target promoter. The results reveal a cellular and molecular mechanism for the nuclear moonlighting of a glycolytic enzyme in plant response to environmental changes.
\end{abstract}

\footnotetext{
${ }^{1}$ Department of Biology, University of Missouri-St. Louis, St. Louis, MO 63121, USA. ${ }^{2}$ Donald Danforth Plant Science Center, St. Louis, MO 63132, USA. ${ }^{3}$ Present address: National Key Laboratory of Crop Genetic and Improvement, College of Plant Science and Technology, Huazhong Agricultural University,

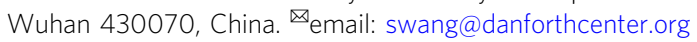


G lyceraldehyde-3-phosphate dehydrogenase (GAPDH) is a glycolytic enzyme converting glyceraldehyde-3-phopshate to 1,3-bisphosphoglycerate. Glycolysis breaks down carbohydrates, providing intermediates for energy production and cellular metabolism, including the synthesis of fatty acids, amino acids, hormones, and osmolytes for drought and salinity protection. Arabidopsis has eight members of GAPDH, two of which are cytosolic forms GAPC1 and GAPC2 ${ }^{1,2}$. Increasing evidence suggests that GAPC also plays important roles in mediating plant response to abiotic and biotic stresses ${ }^{3,4}$. For example, GAPC is involved in plant response to oxidative stress that could result from abiotic and biotic challenges ${ }^{5}$. GAPC has been implicated in plant response to stresses, including cadmium, long-chain bases (dihydrosphingosine), reactive oxygen species, the lipid mediator phosphatidic acid (PA), and cold-induced sweetening and apical dominance of potato ${ }^{6-10}$. We showed that GAPC was involved in mediating plant water loss and Arabidopsis mutants deficient in GAPC1 and GAPC2 suffered a higher transpirational water loss than wild type plants ${ }^{2}$. GAPC affected multiple plant immune responses to bacterial pathogen, such as reactive oxygen species production, programmed cell death, and autophagy ${ }^{8,11}$. GAPC was also involved in viral infection ${ }^{10,12}$.

One mechanism for the GAPC's action in stress response is its stress-induced nuclear translocation. A small pool of GAPC accumulated in the nucleus in Arabidopsis response to treatments with cadmium, bacterial flagellin, PA, and hydrogen sulfide ${ }^{6-8,13,14}$. The nuclear accumulation of GAPC was also observed in tobacco BY-2 (bright-yellow 2) cells exposed to long-chain bases, regulators for programmed cell death in plants ${ }^{15}$. Since GAPC has no nuclear localization signal, post-translational modifications of specific amino acid residues are believed to be important for the stressinduced intracellular translocation. Under certain stress conditions, the highly reactive catalytic cysteine of GAPC undergoes thiol modifications, such as $S$-nitrosylation, $S$-sulfhydration, and $S$ glutathionylation ${ }^{13,14}$ whereas specific lysines can be acetylated to promote nuclear translocation ${ }^{16}$. In addition, lysine ubiquitination by an E3 ubiquitin-ligase was reported as a potential mechanism for GAPC nuclear localization ${ }^{17}$.

Even though some of the mechanisms required for stressinduced nuclear localization is now determined, a clear role of nuclear GAPC remains elusive in plant stress responses. In animal cells, nuclear GAPDH affects transcriptional activity, DNA replication and repair, and epigenetic modifications by binding to various nuclear components, including transcriptional machinery and nucleic acids ${ }^{18,19}$. Because of its potential implication in diverse cellular phenomena, GAPDH is currently being investigated in context with human disorders, including neurodegenerative diseases (e.g. Alzheimer's disease) and cancer ${ }^{20-23}$. In plants, one report indicated that GAPC directly interacted with a DNA sequence encoding NADP-dependent malate dehydrogenase, but the effect of such binding is unknown ${ }^{13}$. Rice GAPC1 was found to bind to the promoters of some glycolytic genes including GAPC1 itself, indicating that GAPC is a transcriptional activator of glycolytic function ${ }^{16}$. This study was undertaken to determine how GAPC affected nuclear function in plant stress responses. Here we show that GAPC interacts with the transcription factor nuclear factor Y subunit C10 (NF-YC10) and regulates transcriptional and physiological responses to heat stress.

\section{Results}

NF-YC10 is identified as a GAPC-binding transcription factor. The nuclear translocation of GAPC under stress raises a possibility that GAPC may play a role in stress-responsive gene expression by modulating transcriptional activity of transcription factor(s) through direct protein-protein interaction. To test this possibility, we screened an Arabidopsis transcription factor library for transcription factors potentially binding to and modulated by GAPC. We modified an Arabidopsis cDNA library composed of $\sim 1500$ transcription factors ${ }^{24}$ to produce recombinant proteins in Escherichia coli. After a library-efficiency transformation, colonies were pooled together, from which the recombinant proteins were isolated as a mixture. A large number of different proteins expressed in the $E$. coli clones was verified by separation of the proteins on a polyacrylamide gel (Fig. 1a). The protein mixture was then co-immunoprecipitated with GAPC2Flag that was purified from Arabidopsis overexpressing the recombinant protein or from control plants with empty vector (EV) using an anti-Flag antibody. SDS-PAGE analysis revealed the successful immunoprecipitation of GAPC2 as determined by the clear GAPC2 band (and immunoglobulin G heavy/light chain bands; Fig. 1b). To identify proteins co-immunoprecipitated with GAPC2, we sequenced the entire immunoprecipitants by mass spectrometry and compared the identified proteins between the GAPC2 sample and EV control. The mass spectrometry-based protein sequencing identified the nuclear factor $\mathrm{Y}$ subunit $\mathrm{C} 10$ (NF-YC10) co-precipitated specifically with GAPC2 (Fig. 1c).

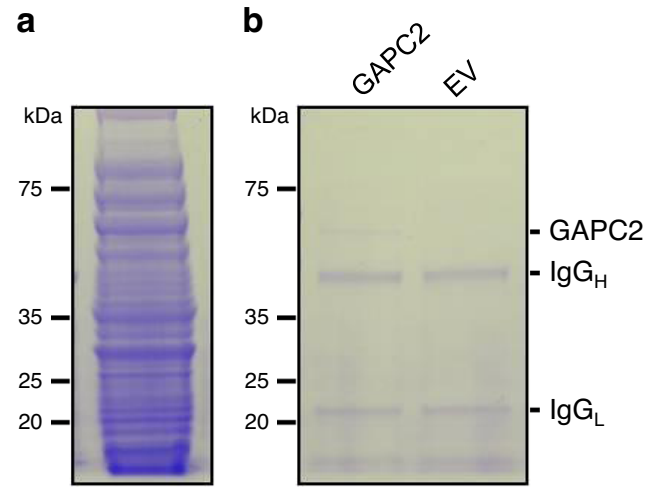

C

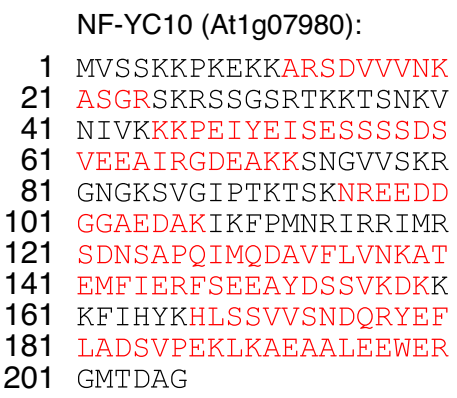

Fig. 1 Screening of Arabidopsis transcription factors to identify GAPC-binding proteins. a Mixture of the purified transcription factors. Proteins were purified by affinity chromatography and separated on a polyacrylamide gel. The gel was stained with Coomassie Brilliant Blue. Protein marker size is on the left. b Gel image of co-immunoprecipitation. Transcription factors co-immunoprecipitated with GAPC2-Flag or empty vector control (EV) were separated on a polyacrylamide gel and stained with Coomassie Brilliant Blue. Protein marker size is on the left. Positions of GAPC2, immunoglobulin G (IgG) heavy and light chains are on the right. c Mass spectrometry-based identification of NF-YC10. Trypsin-digested peptides from the immunoprecipitated samples were sequenced by LC-MS/MS. Shown here is NF-YC10 sequence with the unique peptides in red that were identified with probability $>99 \%$. 
a

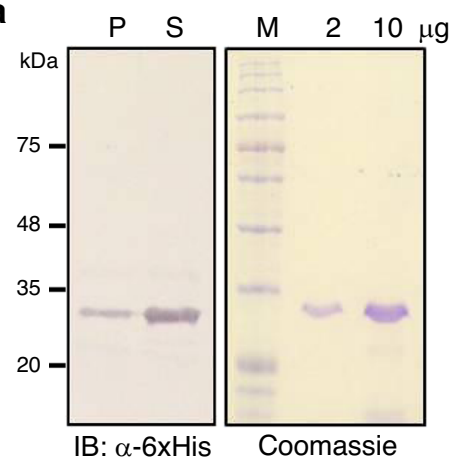

b

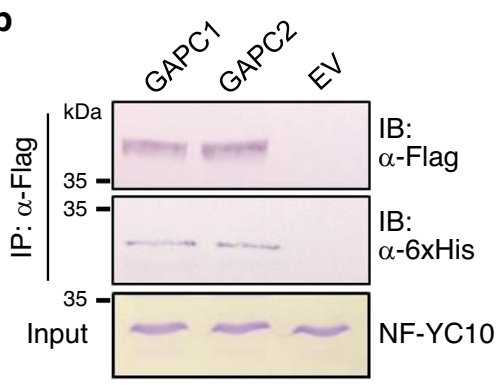

c
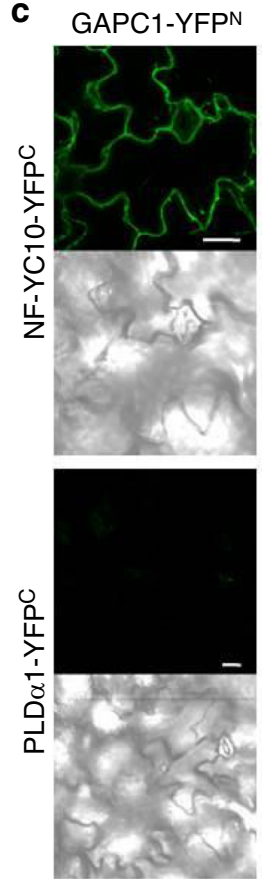
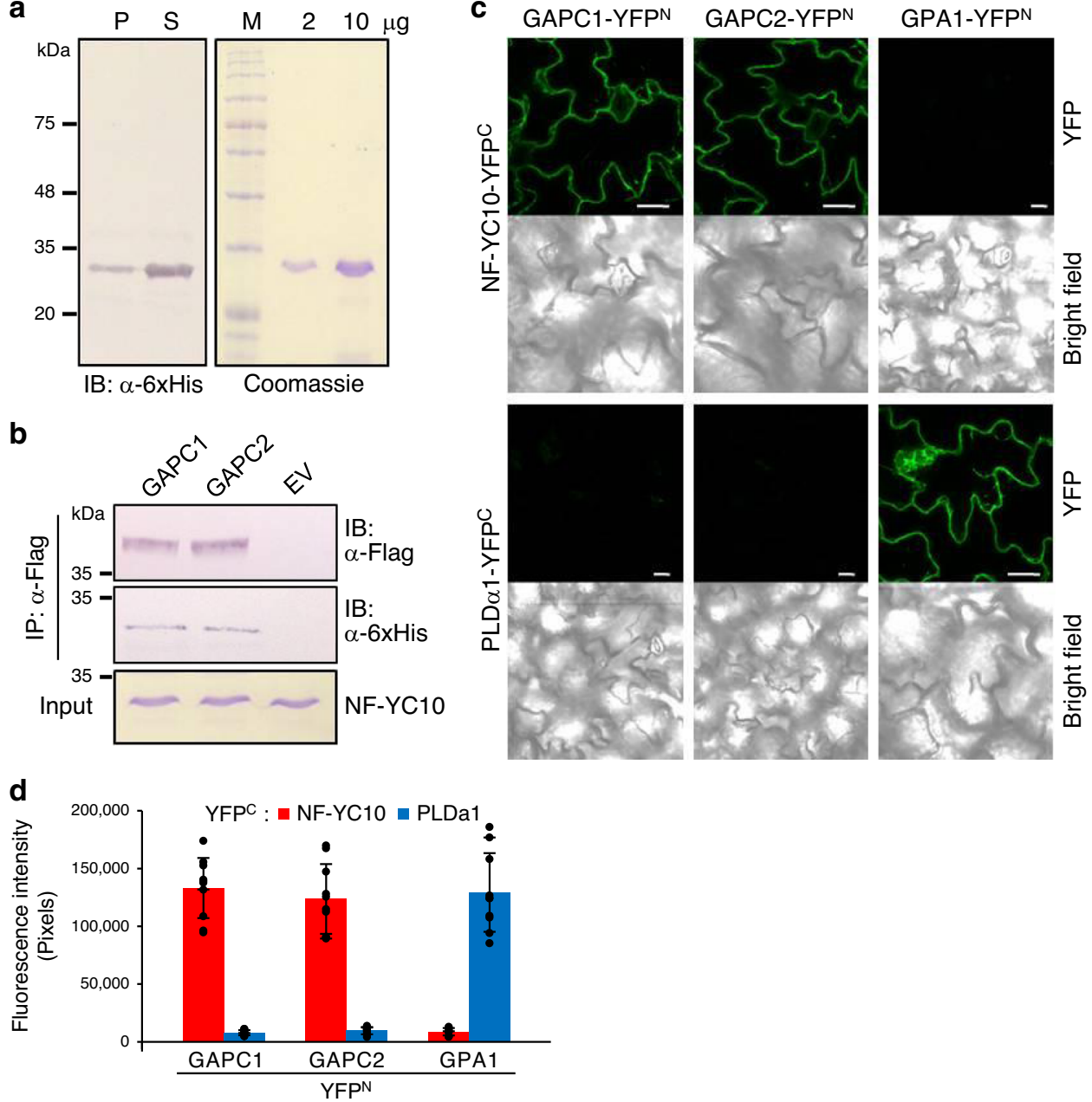

Fig. 2 Physical interaction of GAPC with NF-YC10. a Expression and purification of recombinant NF-YC10 produced in E. coli. Proteins from pellet (P) or supernatant (S) following centrifugation of $E$. coli cell lysate were separated, and NF-YC10-6xHis was probed by immunoblotting (IB) with an anti-6xHis antibody (left panel). Protein marker size is on the left. NF-YC10 was nickel affinity-purified from the E. coli and shown here on polyacrylamide gel stained with Coomassie blue (right panel). M, marker. b Co-immunoprecipitation of GAPC and NF-YC10. GAPC1-Flag, GAPC2-Flag, or empty vector control (EV) was mixed with NF-YC10-6xHis. Immunoprecipitation (IP) was performed using an anti-Flag antibody, then immunoblotting (IB) was performed using the antibodies indicated on the right. NF-YC10 protein input is shown on polyacrylamide gel stained with Coomassie blue (bottom panel). c Bimolecular fluorescence complementation (BiFC) analysis. GAPC-YFPN and NF-YC10-YFPC were transiently co-expressed in tobacco leaves and observed under a confocal microscope. GPA1 and PLD 1 that bound each other were used as controls. Scale bars $=10 \mu \mathrm{m}$. $\mathbf{d}$ Quantification of BiFC. Fluorescence intensity of tobacco leaves used in $\mathbf{c}$ was measured by ImageJ software. Values are average \pm S.D. from 10 randomly chosen regions of tobacco leaves infiltrated. Black dots represent individual data points.

GAPC interaction with NF-YC10 occurs in vitro and in planta. To verify the interaction between GAPC and NF-YC10, we cloned NF-YC10 from Arabidopsis and expressed the recombinant protein as a fusion with $6 \mathrm{xHis}$ tag in E. coli. The recombinant NF-YC10 was present mostly in the soluble fraction of $E$. coli cell lysate and purified to near homogeneity (Fig. 2a). The purified NF-YC10 was then co-immunoprecipitated using an anti-Flag antibody with GAPC1-Flag or GAPC2-Flag purified from Arabidopsis overexpressing the respective proteins or proteins purified from control plants with empty vector (EV). Immunoblotting analysis using an anti-6xHis antibody demonstrated that NF-YC10 was co-precipitated with both GAPC1 and GAPC2, but not with EV control (Fig. 2b). Next, we performed a bimolecular fluorescence complementation (BiFC) assay to verify the GAPC-NF-YC10 interaction in planta. GAPC1 or GAPC2 fused with the $\mathrm{N}$-terminal half of yellow fluorescence protein $($ GAPC-YFPN) and NF-YC10 with C-terminal half of YFP (NF$\mathrm{YC} 10-\mathrm{YFP}^{\mathrm{C}}$ ) were co-expressed transiently in tobacco leaves, and the fluorescent signal was observed under a confocal microscope. For both GAPC1 and GAPC2, co-expression of GAPC and NFYC10 produced detectable fluorescence in the tobacco leaves, but the signal was not observed when GAPC was replaced by a nonNF-YC10-binding protein the Ga subunit of heterotrimeric protein (GPA1; Fig. 2c). Likewise, no fluorescent signal was detected when NF-YC10 was replaced by phospholipase Da1 (PLDa1; Fig. 2c). As GPA1 and PLDa1 interact with each other ${ }^{25,26}$, fluorescence was detected upon their co-expression (Fig. 2c). The fluorescent signal from co-expression of GAPC and NF-YC10 was quantified to be markedly greater than when a counterpart was replaced by GPA1 or PLDa1 (Fig. 2d). Taken together, these results indicate that the GAPC-NF-YC10 interaction occurs both in vitro and in vivo.

GAPC modulates heat tolerance in Arabidopsis. NF-YC10 is involved in heat stress response; its overexpression enhanced the 

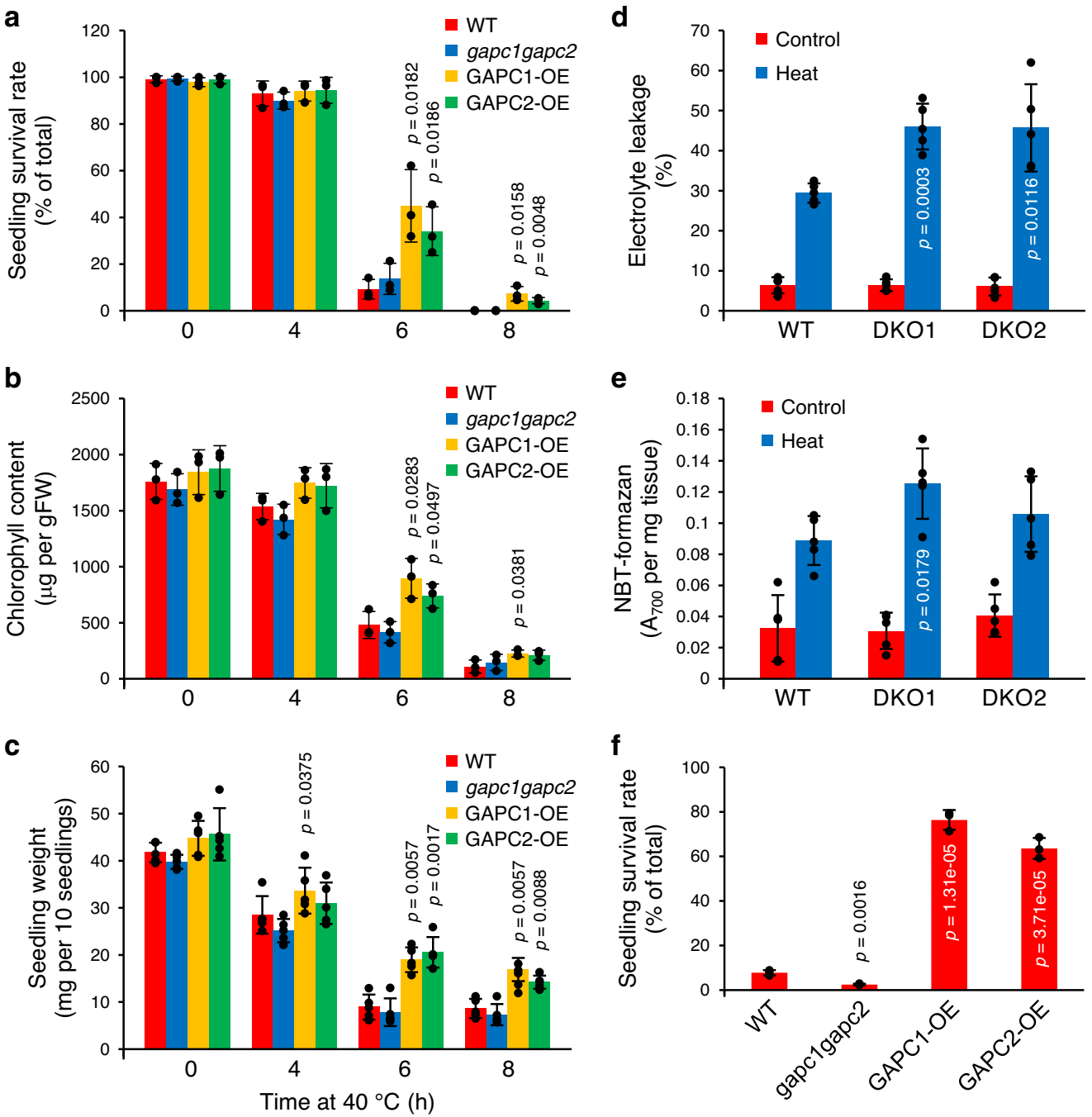

Fig. 3 Phenotypic response of GAPC-altered Arabidopsis to heat. a-c Quantitative measurements of growth response to heat. Five-day-old seedlings were treated at $40^{\circ} \mathrm{C}$ for the indicated times, then placed back to normal temperature condition. Plants were measured for seedling survival rate (a), chlorophyll content (b), and seedling weight (c). Values are average \pm S.D. P values indicate significant difference from WT determined by two-tailed student's $t$ test ( $n=3$ for $\mathbf{a} \& \mathbf{b}$ and 5 for $\mathbf{c}$ independent groups of $>30$ seedlings). Black dots represent individual data points. FW, fresh weight. d Electrolyte leakage. Conductivity was measured with rosette leaves from 3-week-old plants immediately after heat treatment at $40{ }^{\circ} \mathrm{C}$ for $6 \mathrm{~h}$. Electrolyte leakage was calculated and shown here as \% conductivity of total ions (boiled sample). Values are average \pm S.D. $P$ values indicate significant difference from WT determined by two-tailed student's $t$ test $(n=5$ leaves from independent plants). Black dots represent individual data points. DKO, double knockout mutant of GAPC. e Reactive oxygen species production. Rosette leaves from 3-week-old plants were incubated with NBT immediately after heat treatment at $40{ }^{\circ} \mathrm{C}$ for $6 \mathrm{~h}$. NBT-formazan was quantified as absorbance at $700 \mathrm{~nm}$ and shown here as per tissue weight. Values are average \pm S.D. $P$ values indicate significant difference from WT determined by two-tailed student's $t$ test ( $n=5$ leaves from independent plants). Black dots represent individual data points. f Seedling survival at $45^{\circ} \mathrm{C}$. Five-day-old seedlings were treated at $45^{\circ} \mathrm{C}$ for $2.5 \mathrm{~h}$, then placed back to normal temperature condition. After 2 days the seedlings were measured for survival rate. Values are average \pm S.D. $P$ values indicate significant difference from WT determined by two-tailed student's $t$ test ( $n=3$ independent groups of $>30$ seedlings). Black dots represent individual data points.

expression of some heat-inducible genes and rendered Arabidopsis seedlings more tolerant to heat stress, while knockdown mutation of NF-YC10 had the opposite effects ${ }^{27}$. Thus, we tested the effect of altered GAPC expression on plant response to heat stress, using a double knockout mutant gapclgapc2 and plants overexpressing GAPC1 or GAPC2 under the control of the CaMV-35S promoter (GAPC1-OE and GAPC2-OE) that we previously generated and characterized ${ }^{2,28}$. Those alterations of GAPC1 or GAPC2 had no effect on seed germination and seedling growth under normal growth condition (Supplementary Fig. 1A) 7. When 5-day-old seedlings were treated at $40^{\circ} \mathrm{C}$ for varied durations up to $8 \mathrm{~h}$ and then transferred back to the normal temperature conditions for recovery for 2 days, all plants showed no observable difference up to $4 \mathrm{~h}$ of heat stress (Fig. $3 \mathrm{a}-\mathrm{c}$; Supplementary Fig. 1B). However, after $6 \mathrm{~h}$ of heat stress, some of the GAPC-OE seedlings survived whereas most WT and gapclgapc2 seedlings died as indicated by cotyledon bleaching (Supplementary Fig. 1B). Quantitative measurements performed with groups of seedlings indicated that GAPC1-OE and GAPC2-OE, when compared to WT, began to show increased tolerance to heat stress at $6 \mathrm{~h}$ of heat treatment in terms of seedling survival rate, chlorophyll content, and seedling weight (Fig. $3 \mathrm{a}-\mathrm{c}$ ). Although WT and gapclgacp2 seedlings were comparable in growth at $40^{\circ}$ C, gapclgacp2 seedlings displayed increased electrolyte leakage and reactive oxygen species production (Fig. 3d,e), indicating that plants without GAPCs suffer more severe cell damage than WT 

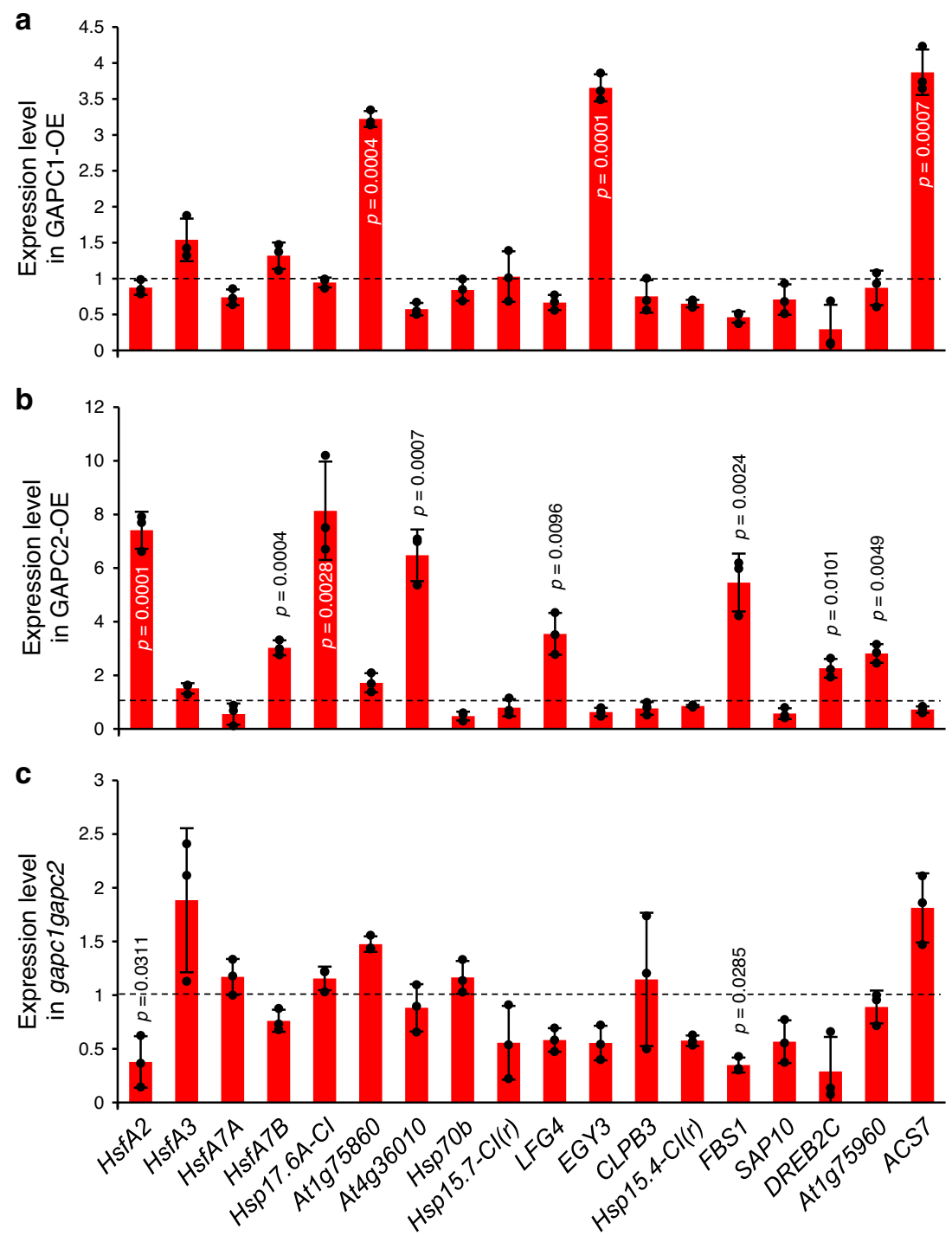

Fig. 4 Expression of heat-inducible genes in GAPC-altered Arabidopsis. Total RNA was extracted from 5-day-old seedlings of GAPC1-OE (a), GAPC2-OE (b), and gapclgapc2 (c) treated at $37^{\circ} \mathrm{C}$ for $5 \mathrm{~h}$ and quantitative RT-PCR was performed with gene-specific primers. Values are average \pm S.D. and shown as fold change to WT (dashed line). $P$ values indicate significant difference from WT determined by two-tailed student's $t$ test ( $n=3$ independent groups of $>10$ seedlings). Black dots represent individual data points. Hsf, heat shock transcription factor; Hsp, heat shock protein; At1g75860, DNA ligase; At4g36010, pathogenesis-related thaumatin superfamily protein; LFG4, LIFEGUARD4 (Bax inhibitor-1 family protein); EGY3, ETHYLENE-DEPENDENT GRAVITROPISM-DEFICIENT AND YELLOW-GREEN-LIKE3; CLPB3, CASEIN LYTIC PROTEINASE B3; FBS1, F-BOX STRESS INDUCED1; SAP10, STRESSASSOCIATED PROTEIN10; DREB2C, DEHYDRATION-RESPONSIVE ELEMENT BINDING PROTEIN 2C; At1g75960, AMP-dependent synthetase and ligase family protein; ACS7, 1-AMINO-CYCLOPROPANE-1-CARBOXYLATE SYNTHASE7.

under heat stress. The difference in heat tolerance between $G A P C$-altered and WT plants was more obvious when they were subjected to a higher temperature. When 5-day-old seedlings were treated at $45^{\circ} \mathrm{C}$ for $2.5 \mathrm{~h}$ then returned to normal temperature conditions, more than $60 \%$ of GAPC-OE seedlings remained alive, whereas only $8 \%$ of WT survived and nearly all gapclgacp2 seedlings died (Fig. 3f). Taken together, the data suggest that the presence of GAPC is positively associated with heat tolerance in Arabidopsis.

GAPC regulates the expression of heat-inducible genes. NFYC10 overepxression enhances the expression of some heatinducible genes ${ }^{27}$. To determine if these NF-YC10 target genes are affected by GAPC alterations, we tested GAPC-OEs and gapclgapc2 for heat-induced expression of 18 heat-inducible genes that were up-regulated by NF-YC10. GAPC1 and GAPC2 overexpression increased the transcript levels of different subsets of the heat-inducible genes over those of WT (Fig. 4a, b). GAPC1OE seedlings exhibited an $\sim 3$ - to 4-fold increase in At1g75860, EGY3 (ETHYLENE-DEPENDENT GRAVITROPISM-DEFICIENT AND YELLOW-GREEN-LIKE3), and ACS7 (1-AMINO-CYCLOPROPANE-1-CARBOXYLATE SYNTHASE7) under heat stress, compared to those levels in WT (Fig. 4a). By comparison, GAPC2-OE seedlings had increased transcript levels up to 8-fold of 8 heat-inducible genes, HsfA2 (heat shock transcription factor A2), HsfA7B, Hsp17.6A-CI, At4g36010 (encoding a pathogenesisrelated thaumatin superfamily protein), LFG4 (LIFEGUARD4, a Bax inhibitor1 family protein), FBS1 (F-BOX STRESS INDUCED1), DREB2C (DEHYDRATION-RESPONSIVE ELEMENT BINDING PROTEIN 2C), and At1g75960 (encoding an AMP-dependent synthetase and ligase family protein) (Fig. 4b). A slight decrease in the expression of $H s f A 2$ and $F B S 1$ was observed in gapclgapc2 (Fig. 4c). Expression level of GAPC was not significantly affected by overexpression of the other GAPC, and NF- 
a

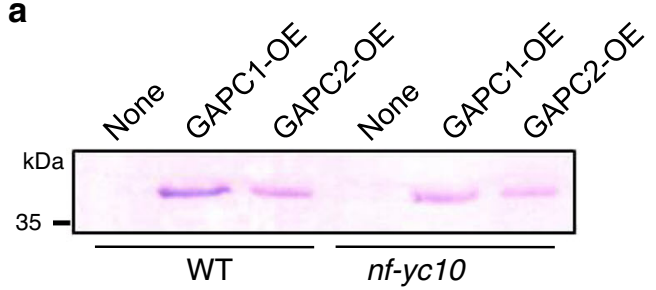

b

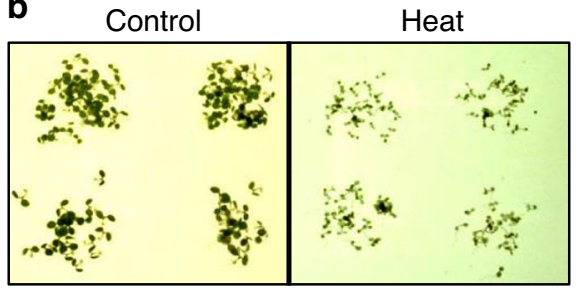

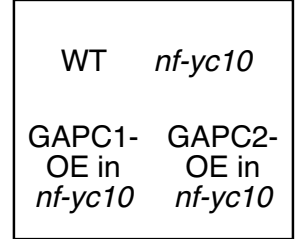

GAPC1- GAPC2$\underset{n f-y c 10}{\mathrm{OE} \text { in }} \mathrm{OE}$ in
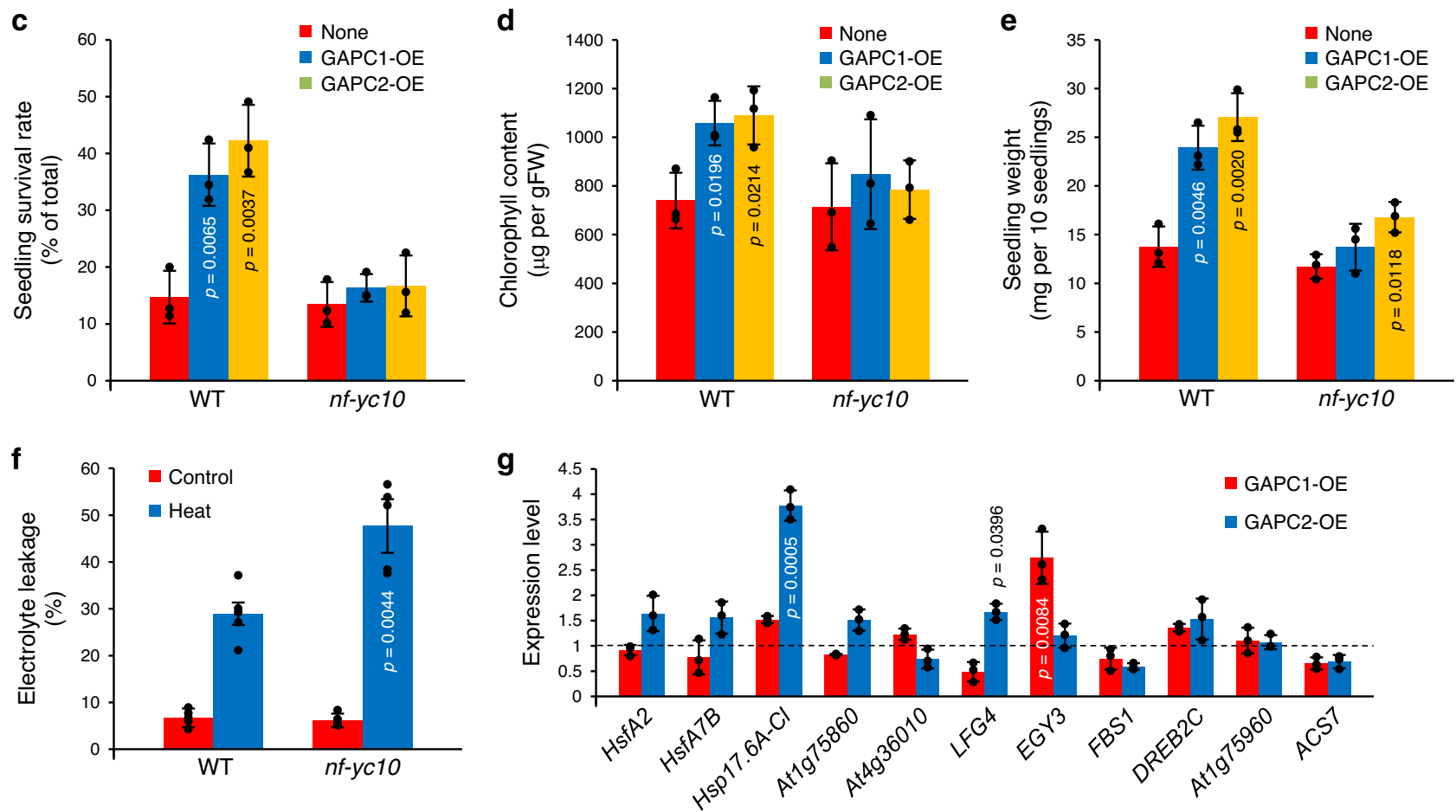

Fig. 5 Effects of GAPC overexpression in $\mathbf{n f - y c 1 0}$ on heat response. a Immunoblotting of GAPC. Total proteins were extracted from 5-day-old transgenic seedlings overexpressing GAPC-Flag as indicated on the top in the background plants indicated at the bottom. GAPC was probed with an anti-Flag antibody by immunoblotting. b Representative images of heat-treated plants. Five-day-old seedlings were untreated (Control) or treated at $40{ }^{\circ} \mathrm{C}$ for $6 \mathrm{~h}$, then placed back to normal temperature condition. Images were taken after 2 days of heat shock. c-e Quantitative measurements of heat response. Plants with genetic background indicated on the $\mathrm{x}$-axis were treated at $40^{\circ} \mathrm{C}$ for $6 \mathrm{~h}$ and measured for seedling survival rate (c), chlorophyll content (d), and seedling weight (e). Values are average \pm S.D. $P$ values indicate significant difference from background line (None) determined by two-tailed student's $t$ test $(n=3$ independent groups of $>30$ seedlings). Black dots represent individual data points. FW, fresh weight. $\mathbf{f}$ Electrolyte leakage of WT and $n f-y c 10$. Conductivity was measured with rosette leaves from 3-week-old plants immediately after heat treatment at $40^{\circ} \mathrm{C}$ for $6 \mathrm{~h}$. Electrolyte leakage was calculated and shown here as $\%$ conductivity of total ions (boiled sample). Values are average \pm S.D. P values indicate significant difference from WT determined by two-tailed student's $t$ test ( $n=5$ leaves from independent plants). Black dots represent individual data points. $\mathbf{g}$ Expression of heat-inducible genes. Total RNA was extracted from 5-day-old seedlings treated at $37^{\circ} \mathrm{C}$ for $5 \mathrm{~h}$ and quantitative RT-PCR was performed with gene-specific primers. Values are average \pm S.D. and shown as fold change to background line (nf-yc10; dashed line). $P$ values indicate significant difference from nf-yc10 determined by two-tailed student's $t$ test ( $n=3$ independent groups of $>10$ seedlings). Black dots represent individual data points.

YC10 expression level remained similar in all GAPC-altered plants (Supplementary Fig. 2). The expression of GAPC1 and GAPC2 was not induced by heat stress at $38^{\circ} \mathrm{C}$ for up to $3 \mathrm{~h}$, as indicated by the data obtained from public microarray database (AtGenExpress) ${ }^{29}$.

The GAPC-mediated heat responses require NF-YC10. To determine if the up-regulation of heat-inducible genes and the enhanced heat tolerance observed in GAPC-OE are dependent on the presence of NF-YC10, we overexpressed GAPC in NF-YC10 knockout mutant $(n f-y c 10)$ and tested the transgenic plants for the expression levels of the 11 heat-inducible genes up-regulated by GAPC overexpression in WT and growth phenotype in response to heat. Protein abundance increased by GAPC overexpression in both WT and $n f-y c 10$ backgrounds was confirmed by immunoblotting (Fig. 5a). The GAPC-OE in $n f-y c 10$ (GAPC-
$\left.\mathrm{OE}_{n f-y c 10}\right)$ plants exhibited growth phenotype indistinguishable from their background plant $n f-y c 10$ under heat stress (Fig. 5b). Seedling survival rate, chlorophyll content, and seedling weight were not significantly increased by GAPC overexpression in $n f$ $y c 10$ at $40^{\circ} \mathrm{C}$ for $6 \mathrm{~h}$, while still increased by GAPC overexpressed in WT (GAPC-OE $\mathrm{WT}_{\mathrm{WT}}$ Fig. 5c-e). When compared to WT, $n f-y c 10$ showed an increase in heat-induced electrolyte leakage as did gapclgapc2 seedlings at $40^{\circ} \mathrm{C}$ (Fig. 5f). The GAPC-OE ${ }_{n f-y c 10}$ plants, when compared with the $n f-y c 10$ plants, showed no or substantially attenuated heat induction of the genes that were highly up-regulated in GAPC-OE ${ }_{\mathrm{WT}}$ (Fig. 5g). GAPC1-OE seedlings showed no increased transcript levels of At1g75860 and $A C S 7$ that were up-regulated in GAPC1-OE $\mathrm{WT}_{\mathrm{WT}}$ under heat stress (Fig. 4a vs. Fig. 5g). The transcript level of EGY3 was increased in GAPC1-OE $\mathrm{E}_{n-y c 10}$ but the magnitude of increase was smaller than that in GAPC1-OE $\mathrm{WT}_{\mathrm{WT}}$, compared to their respective background 
$n f-y c 10$ and WT seedlings (Fig. $4 \mathrm{a}$ vs. Fig. 5g). GAPC2-OE ${ }_{n f-y c 10}$ seedlings had no increased transcript level of 6 genes up-regulated in GAPC2-OE $\mathrm{WT}_{\mathrm{WT}}$ under heat stress (Fig. $4 \mathrm{~b}$ vs. Fig. 5g). The magnitude of increase in Hsp17.6A-CI and LFG4 transcripts was $\sim 2$-fold less than that in GAPC2-OE ${ }_{\mathrm{WT}}$ (Fig. 4b vs. Fig. 5g). Taken together, the results suggest that the GAPC effect on heat tolerance requires the presence of NF-YC10, and that NF-YC10 may function as a downstream target of GAPC in the signaling pathway for plant response to heat stress.

Heat stress promotes GAPC accumulation in nuclei. We then tested whether, in response to heat, the cytosolic GAPCs might enter the nucleus where they could interact with the transcription factor NF-YC10. Arabidopsis transgenic lines overexpressing GAPC1 or GAPC2 fused with the green fluorescence protein (GAPC-GFP) were generated to observe their subcellular location. Under normal laboratory temperatures $\left(23^{\circ} \mathrm{C}\right)$, the fluorescence signal was observed mostly in the cytosol for both GAPC1 and GAPC2 (Fig. 6a, Control). After heat treatment at $40^{\circ} \mathrm{C}$ for $6 \mathrm{~h}$, however, significant GFP signal was detected in the nucleus of many leaf epidermal cells for both GAPC1 and GAPC2 (Fig. 6a, Heat and 6b). The association of GAPC-GFP signal with the nucleus was confirmed by counterstaining with the nucleic acid-specific 4',6-diamidino-2-phenylindole (DAPI; Fig. 6c). Nuclear accumulation of other cytosolic glycolytic enzymes, such as non-phosphorylating GAPDH (NP-GAPDH) and hexokinase 1 (HXK1), was not observed in heat-treated transgenic seedlings overexpressing those as a GFP-fusion protein (Supplementary Fig. 3). The heat-induced accumulation of GAPC in the nucleus was also observed with nuclear proteins extracted from heattreated GAPC-OE plants followed by immunoblotting analysis. GAPC1 and GAPC2 proteins were detected in the cytosol regardless of heat treatment, whereas they were found in the nucleus only upon heat stress (Fig. 6d, e). Successful separation of the two fractions was confirmed by probing organelle-specific marker proteins (PEPC for cytosol and histone $\mathrm{H} 3$ for nucleus; Fig. 6d).

To test whether endogenous GAPC displays heat-induced nuclear localization, we isolated nuclei from heat-treated WT Arabidopsis and performed immunoblotting using a GAPCspecific antibody. GAPC was present in the nuclei isolated from heat-treated plants, but not in the nuclei from untreated control (Fig. 6f). The antibody was specific to GAPCs because it detected the GAPC band from WT but not from gapclgapc2 protein extracts (Fig. 6g). Those results indicate that heat stress promotes the nuclear translocation of endogenous GAPC protein molecules from the cytosol.

The GAPC-mediated responses require its nuclear accumulation. We next decided to see if interference with the GAPC nuclear localization compromises its ability to control the heat tolerance of seedlings and the expression of heat-inducible genes. While GAPDH, including GAPCs, has no nuclear localization sequence, human GAPDH (HsGAPDH) has three lysine residues (K117, K227, and K251) whose acetylation is necessary and sufficient for apoptotic stress-induced nuclear translocation of $\mathrm{HsGAPDH}^{30}$. The three lysines are conserved in Arabidopsis GAPC at K121, K231, and K255 (Fig. 7a). In addition, GAPC2 was reported to be acetylated at four lysine residues (K130, K219, $\mathrm{K} 223$, and K255) when expressed and purified in E. coli ${ }^{31}$. Thus, we substituted several combinations of the six lysines (K255 overlapping) to alanines in both GAPC1 and GAPC2 and overexpressed the various lysine-mutated proteins in Arabidopsis (Fig. 7a). We found that only one combination of the lysine mutations (K121A and K231A; GAPCmut) compromised the heat-induced nuclear accumulation of both GAPC1 and GAPC2, as revealed by immunoblotting of nuclei isolated from the transgenic plants (Fig. 7b; Supplementary Fig. 4A) and confocal microscopic analysis of GFP-fused GAPCmut (Fig. 7c, d). Analyses of seedling survival under heat stress condition and the gene expression profiling demonstrated that when overexpressed and compared to WT, both GAPC1mut and GAPC2mut failed to enhance heat tolerance of growth (Fig. 7e) and increase the expression of some heat-inducible genes that were observed to be up-regulated by the intact GAPC overexpression (Fig. 7f). There was no significant difference in catalytic activity between GAPCmut and intact GAPC (Supplementary Fig. 4B). These data suggest that GAPC is required to translocate into the nucleus to affect NF-YC10-mediated heat responses observed in GAPC$\mathrm{OE}_{\mathrm{WT}}$.

The heat responses require the GAPC and NF-YC10 interaction. The above results of GAPC-enhanced heat responses were obtained mostly from ectopic overexpression of GAPC by the constitutive CaMV-35S promoter. To determine the physiological relevance of these observations in native plants, we generated two independent lines of gapclgapc2 plants genetically complemented with GAPC2 or GAPC2mut under its native promoter, and compared their heat phenotype and the expression of heatinducible genes with those of gapc1gapc2. In addition, to test whether the GAPC effect is dependent on the GAPC and NFYC10 interaction in nuclei, we identified a GAPC2mut' that does not bind NF-YC10 but still underwent nuclear translocation under heat stress. The lysine-mutated GAPCmut' protein (K121/ $219 / 223 / 255 A$ ) failed to bind NF-YC10, as indicated by the lack of co-immunoprecipitation (Fig. 8a). Hence, GAPC2mut'-complemented gapclgapc2 was included to examine the effect of GAPC-NF-YC10 interaction, as well as GAPC nuclear accumulation, on the heat responses of gapclgapc2. A significant increase in the expression of GAPC2 and its protein product in all the complemented plants was confirmed by qRT-PCR and immunoblotting, respectively (Fig. $8 \mathrm{~b}$ ). While the heat-induced electrolyte leakage and reactive oxygen species production in GAPC2complemented gapc1gapc2 were fully restored to the level of WT, that of GAPC2mut- or GAPC2mut'-complemented gapc1gapc2 still remained significantly higher than WT (Fig. 8c, d). Likewise, the expression of the two heat-inducible genes observed to be down-regulated in gapclgapc2 (HsfA2 and FBS1; Fig. 4c) was recovered in GAPC2-complemented gapc1gapc2, but not in GAPC2mut- or GAPC2mut'-complemented gapc1gapc2 (Fig. 8e). Restoration of the cytosolic function of GAPC in the complemented plants was confirmed by measuring the catalytic activity of GAPC (Supplementary Fig. 5). Together with the heatinduced nuclear accumulation of endogenous GAPC (Fig. 6f), these data indicate that both GAPC nuclear localization and NFYC10 interaction are required for heat tolerance in native plants, not only upon GAPC overexpression. The full recovery by GAPC2 complementation also verifies that the heat sensitivity observed in gapclgapc2 (Figs. 3 and 4) is due to GAPC disruption.

GAPC enhances DNA binding ability of NF-YC10. The expression of some NF-YC10 target genes increased by GAPC overexpression led us to see whether GAPC could affect the ability of NF-YC10 to bind its target promoters. Sato et al. confirmed experimentally that NF-YC10 bound directly to the promoters of $H s f A 2, H s f A 3$, and $A t 1 g 75860^{27}$. Thus, to verify GAPC effects on DNA binding ability of NF-YC10 to these promoters, we performed chromatin immunoprecipitation (ChIP) using mesophyll protoplasts that were isolated from the GAPC-altered Arabidopsis lines. The protoplasts were transfected with 35S:NF- 

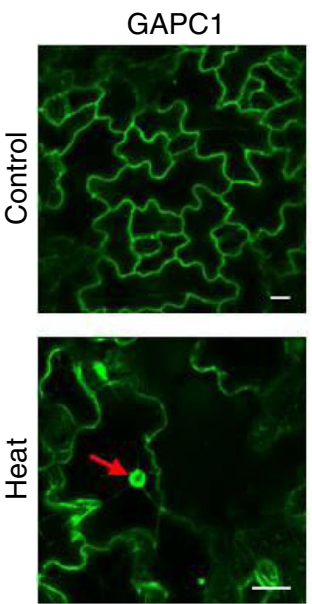

b

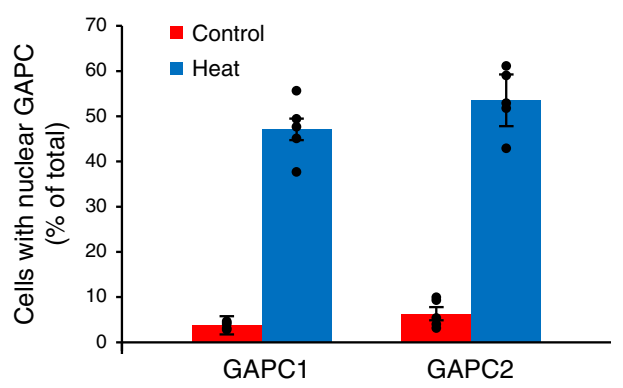

d
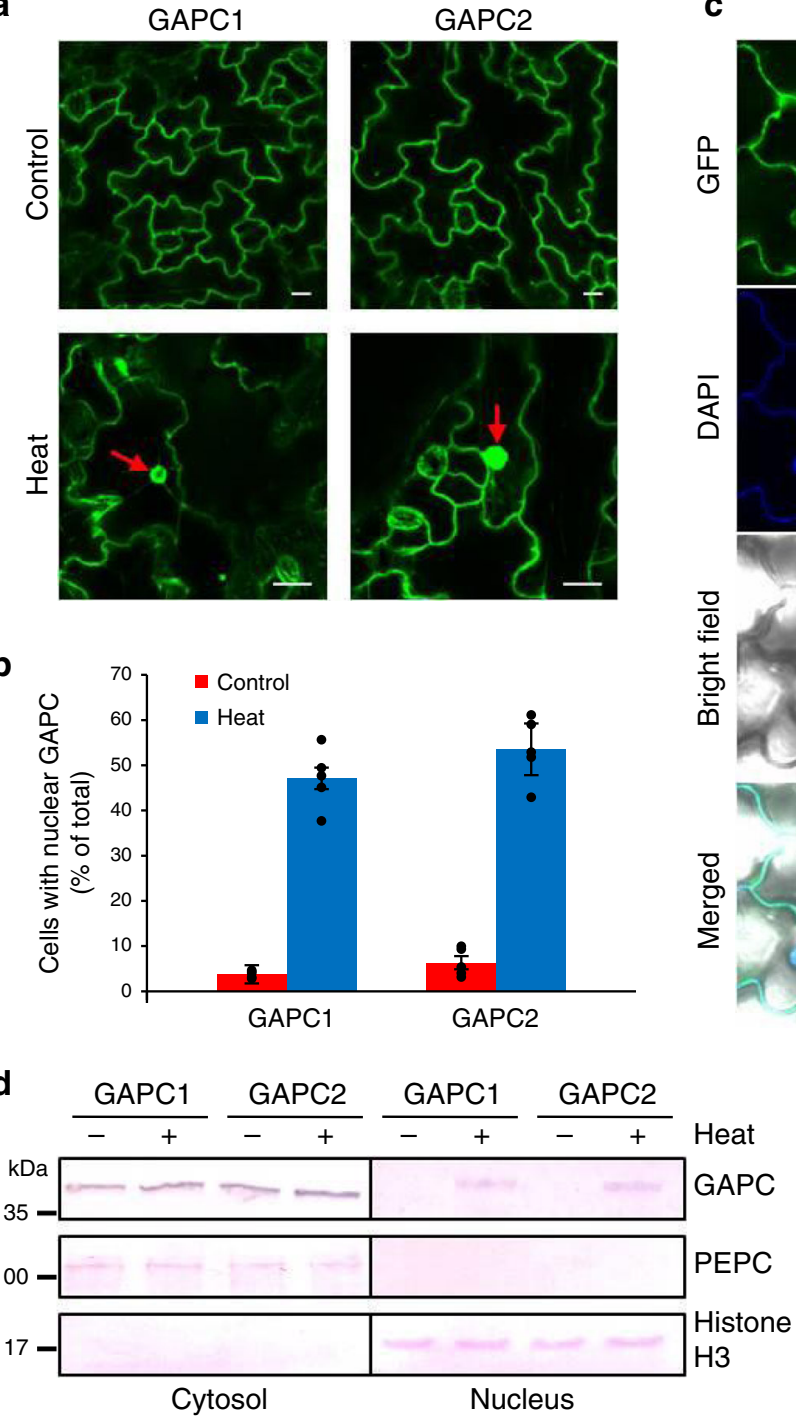

$\frac{0}{\text { ㄴ }}$
C

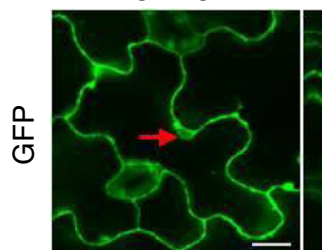

GAPC2
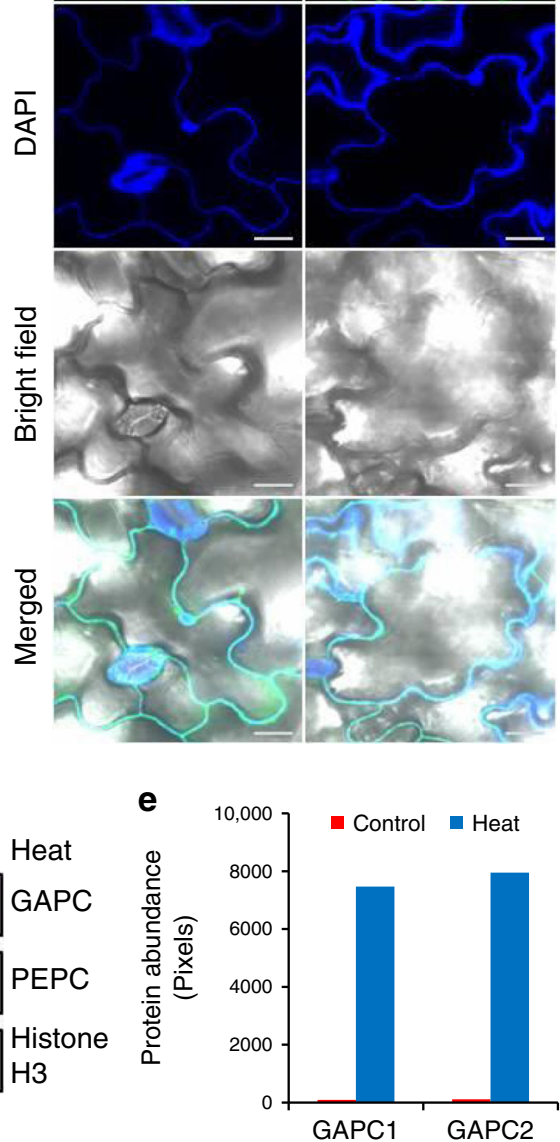

$\mathbf{f}$

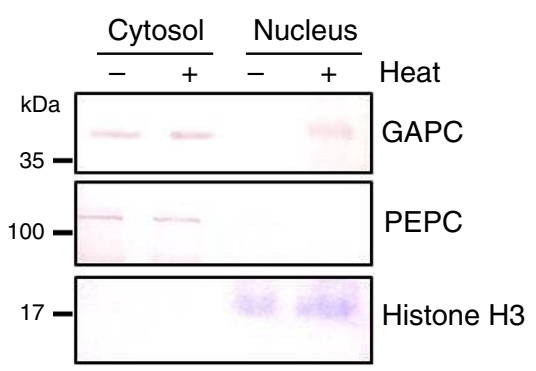

g

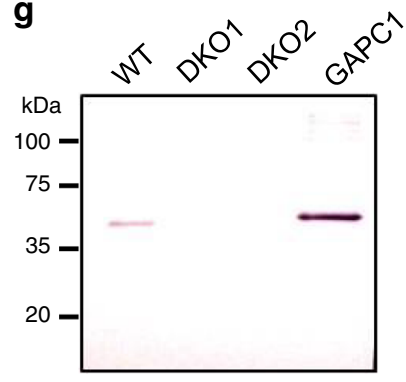

YC10-Flag, then heat-treated at $37^{\circ} \mathrm{C}$ for $5 \mathrm{~h}$. When ChIP was carried out with an anti-Flag antibody, the successful isolation of all three gene's promoter regions associated with NF-YC10 was confirmed by subsequent PCR amplification (Fig. 9a). No PCR product was detected when the antibody was omitted or when WT protoplasts were not transfected (mock; Fig. 9a). A negative control gene (ubiquitin10) showing no DNA band confirmed the specificity of NF-YC10 binding to the heat-inducible genes (Fig. 9a). Quantitative PCR analysis revealed that when compared to those found in WT, HsfA2 and At $1 g 75860$ promoter regions were highly enriched in GAPC2-OE and GAPC1-OE protoplasts respectively, with no significant change in $H s f A 3$ promoter region precipitated with NF-YC10 (Fig. 9b). These results are in agreement with the increased expression of $H s f A 2$ and At1g75860 in GAPC2-OE and GAPC1-OE seedlings, respectively (Fig. 4a, b). We verified by immunoblotting using the anti-Flag antibody that NF-YC10 was expressed comparably among the different samples (Fig. 9c), indicating that the DNA enrichment observed in GAPC$\mathrm{OE}$ protoplasts was independent of NF-YC10 protein abundance.

\section{Discussion}

Enzyme moonlighting is a phenomenon whereby an enzyme can perform secondary non-enzymatic functions acquired through evolution, such as transcriptional regulation, signal transduction, and apoptosis, in addition to its canonical catalytic function ${ }^{19}$. 
Fig. 6 Heat-induced nuclear accumulation of GAPC. a Fluorescence image of Arabidopsis leaf cells. Five-day-old transgenic seedlings overexpressing GAPCGFP were untreated (Control) or treated at $40^{\circ} \mathrm{C}$ for $6 \mathrm{~h}$ and observed under a confocal microscope. Arrows indicate the nucleus. Scale bars $=10 \mu \mathrm{m}$. $\mathbf{b}$ The number of cells with nuclear GAPC. Plants were treated and observed as in $\mathbf{a}$. Cells with clear fluorescence in the nucleus were counted and shown here as \% of total cells counted. Values are average \pm S.D. from 5 leaves independently treated. Black dots represent individual data points. c Confirmation of the nucleus. The heat-treated seedlings were stained with 4',6-diamidino-2-phenylindole (DAPI) for nuclear counterstaining and observed under a confocal microscope. Shown are representative images taken at the same focal plane. Arrows indicate the nucleus. Scale bars $=10 \mu \mathrm{m}$. $\mathbf{d}$ Immununoblotting of GAPC in cytosolic and nuclear fractions. Five-day-old transgenic seedlings overexpressing GAPC-Flag were untreated (-) or treated at $40{ }^{\circ} \mathrm{C}$ for $6 \mathrm{~h}(+)$, and cytosolic and nuclear fractions were separated. GAPC was probed with an anti-Flag antibody by immunoblotting. Phosphoenolpyruvate carboxylase (PEPC) and histone H3 were used as cytosolic and nuclear markers, respectively. e Quantification of nuclear GAPC. Protein band intensity of GAPC in the nuclear fraction in $\mathbf{d}$ was measured by ImageJ software. $\mathbf{f}$ Immununoblotting of endogenous GAPC in cytosolic and nuclear fractions. Five-day-old wild type seedlings were untreated ( - ) or treated at $40^{\circ} \mathrm{C}$ for $6 \mathrm{~h}(+)$, and cytosolic and nuclear fractions were separated. GAPC was probed with an anti-GAPC antibody by immunoblotting. PEPC and histone $\mathrm{H} 3$ were used as cytosolic and nuclear markers, respectively. $\mathbf{g}$ GAPC antibody test for specificity. Total proteins were extracted from 5-day-old seedlings indicated on the top. GAPC was probed with the anti-GAPC antibody used in $\mathbf{f}$ by immunoblotting. Protein marker size is on the left. WT, wild type; DKO, double knockout mutant of GAPC; GAPC1, recombinant GAPC1 purified from E. coli used as a control.

The glycolytic enzyme GAPC is a moonlighting enzyme and plays important roles in plant response to stress, but its role in the nucleus in mediating stress response was unclear. Based on our data presented in this study, we propose that heat stress promotes translocation of a portion of the cytoplasmic GAPC pool into Arabidopsis nuclei, where GAPC is associated with the transcription factor NF-YC10 to increase the expression of target heat-inducible genes and plant heat tolerance (Fig. 10). These findings show that GAPC regulates transcriptional activity by directly interacting with a specific transcription factor in plants.

Nuclear factor Y (NF-Y) is an evolutionarily conserved transcription factor family composed of three subunits, NF-YA, NF$\mathrm{YB}$, and NF-YC, which can form functional heterodimers or heterotrimers with one another in a heat-dependent manner ${ }^{32}$. Plant genomes have multiple members encoding each NF-Y subunit (10 genes for each subunit in Arabidopsis), enabling a wide variety of heteromeric combinations and potentially diverse functions. In addition, NF-Y subunits can also interact with other proteins in various kinds of complexes. Particularly, NF-YC10, also known as DNA POLYMERASE II SUBUNIT B3-1 (DPB3-1), binds to DEHYDRATION RESPONSE ELEMENT-BINDING PROTEIN 2A (DREB2A) and forms a heterotrimer with NF-YA2 and NF-YB3 in a heat stress-dependent manner in Arabidopsis ${ }^{27}$. The heterotrimer forms a transcriptional complex with DREB2A at the promoters of heat-inducible DREB2A target genes, thus enhancing the efficiency of transcription by DREB2A $\mathrm{A}^{27}$. In this regard, GAPC interaction with NF-YC10 might affect the structural integrity and/or regulatory activity of the transcriptional complex to regulate the expression of heat-inducible genes. However, our co-immunoprecipitation assays demonstrated that GAPC neither directly bound to the components of the transcriptional complex, including DREB2A, nor noticeably affected NF-YC10 interaction with the individual binding partners (Supplementary Fig. 6). While these analyses are limited to in vitro conditions, other approaches considering post-translational modification and nuclear elements that might come into play could uncover the effect of GAPC on the NF-Y heterocomplex. Meanwhile, GAPC overexpression increased the expression of other heat-inducible genes that were not DREB2A target genes, as well as the genes downstream of DREB2A. Indeed, the expression of $H s f A 2$, which is not a DREB2A target gene, is increased by NFYC10 overexpression and its promoter is directly bound by NFYC10 under heat stress ${ }^{27}$. This implies that NF-YC10 can enhance the expression of heat-inducible genes through DREB2A-independent pathways as well. These data, together with our results from ChIP assay, suggest that GAPC affects the ability of NF-YC10 itself to bind its target promoters, such as that of HsfA2. Although GAPC-DNA interaction has been reported $^{13,16}$, direct interaction of GAPC with any regulatory elements of the heat-inducible genes can be ruled out by our observation of no or marginal heat induction of the genes in GAPC-OE ${ }_{n f-y c 10}$. Therefore, it should be through the direct interaction with NF-YC10 that GAPC increases the expression of the heat-inducible genes. It should be noted that the transcription factor library screened for GAPC interaction did not contain all transcription factors in Arabidopsis ${ }^{24}$, and thus GAPC may interact with other transcription factors under stress.

Our data show that the presence of GAPC in the nucleus is induced by heat stress. As described earlier, GAPC can undergo different conditional post-translational modifications on its highly reactive cysteine residue, which possibly determines some of its moonlighting functions. Post-translational modification is speculated as a key initial process leading to stress-dependent nuclear translocation of GAPC. S-nitrosylation might be important because $S$-nitrosylation level of GAPC was transiently increased in salt-treated tobacco BY-2 cells, and mutation of the active cysteines into serines impaired salt-dependent GAPC interaction with osmotic stress-activated protein kinase (OSAK) in the nucleus but not in the cytoplasm ${ }^{33}$. However, both cadmium- and long-chain base-induced nuclear translocation of GAPC observed in Arabidopsis and tobacco, respectively, were independent of $S$-nitrosylation ${ }^{6,15}$. A reduced nuclear localization of GAPC was observed in Arabidopsis des1 mutant defective in Lcysteine desulfhydrase DES1 and this was recovered by exogenous sulfide treatment ${ }^{14}$. Together with detection of a sulfhydryl modification of catalytic cysteine in nuclear GAPC $1^{14}$, this suggests that $S$-sulfhydration is a possible mechanism for GAPC nuclear localization. It is reported that oxidative stress by $\mathrm{H}_{2} \mathrm{O}_{2}$ treatment also induces nuclear localization of GAPC by modifying the catalytic cysteine $(\mathrm{C} 156)^{5}$, discrepant with another report that C156S had no effect on GAPC nuclear translocation by cadmium-induced oxidative stress ${ }^{6}$. Thus, different stress signals may differentially modify GAPC on its active site cysteine residues, as observed for those of animal GAPDH.

Interestingly, under apoptotic stressors, acetylation at three lysines (K117, K227, and K251 in HsGAPDH) is required for human GAPDH translocation into the nucleus ${ }^{30}$. The importance of lysine acetylation for stress-induced GAPC nuclear localization was also shown in rice subjected to oxidative stress ${ }^{16}$. However, none of the lysines identified to be responsible for rice GAPC nuclear localization (K57, K74, and K217 in OsGAPDH1) corresponded to those for HsGAPDH ${ }^{16}$. Although the three lysines in HsGAPDH and OsGAPDH1 are absolutely conserved in Arabidopsis GAPC, only one of them (K76 in AtGAPC) is acetylated in unstressed Arabidopsis from which K76, K130, K198, K263, K267, and K310 found to be acetylated ${ }^{34}$. Moreover, our data presented here indicate that two lysines (K121 and K231 in AtGAPC), which are necessary for HsGAPDH nuclear 
a
1 MADKKIRIGI NGFGRIGRLV ARVVLQRDDV ELVAVNDPFI TTEYMTYMFK
51 YDSVHGQWKH HELKVKDDKT LLFGEKPVTV FGIRNPEDIP WGEAGADFVV
101 ESTGVFTDKD KAAAHLKGGA KKVVISAPSK DAPMFVVGVN EHEYKSDLDI
151 VSNASCTTNC LAPLAKVIND RFGIVEGLMT TVHSITATQK TVDGPSMKDW
201 RGGRAASFNI IPSSTGAAKA VGKVLPSLNG KLTGMSFRVP TVDVSVVDLT
251 VRLEKAATYD EIKKAIKEES EGKMKGILGY TEDDVVSTDF VGDNRSSIFD
301 AKAGIALSDK FVKLVSWYDN EWGYSSRVVD LIVHMSKA
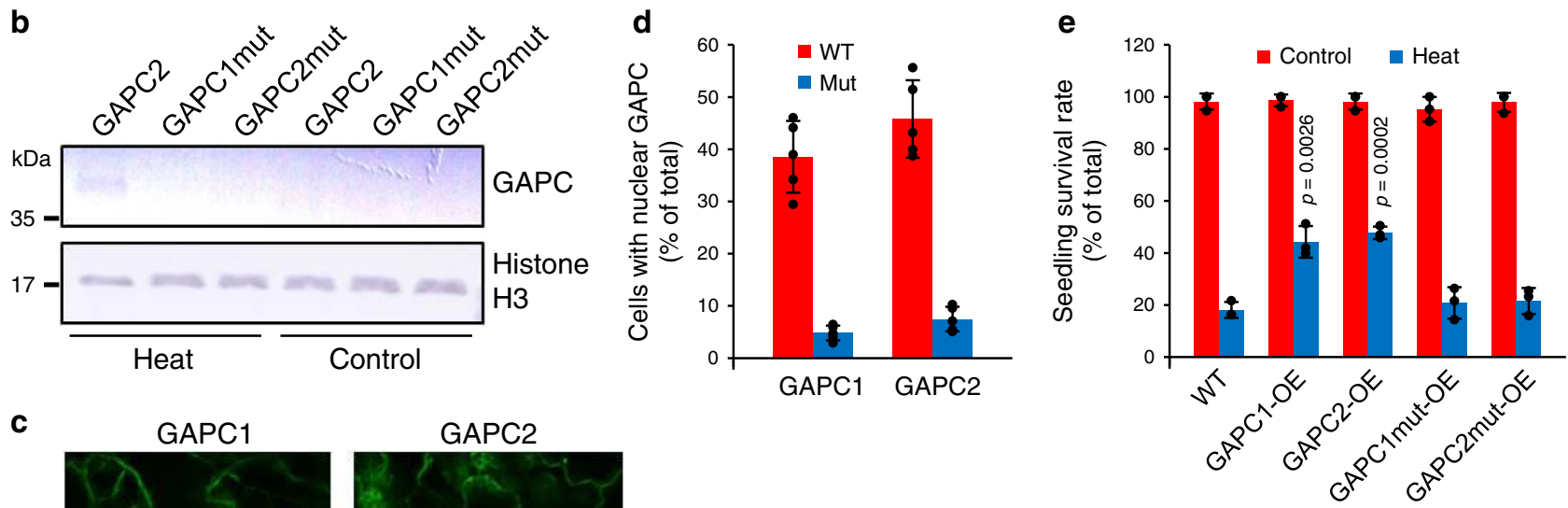

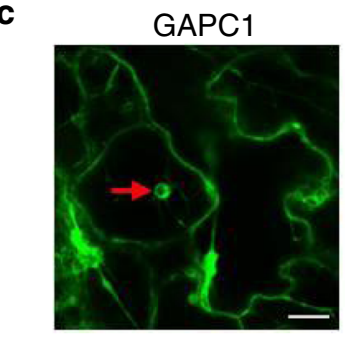

GAPC1mut

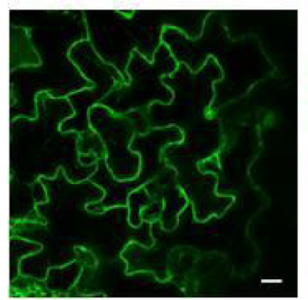

GAPC2

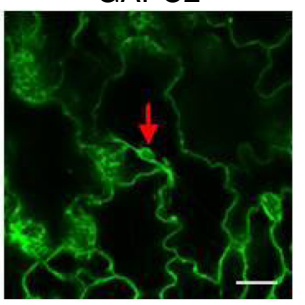

GAPC2mut

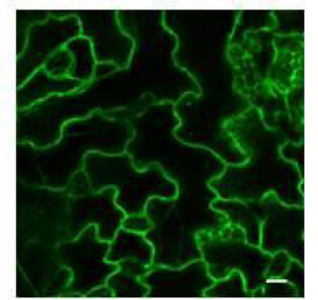

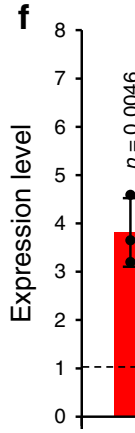
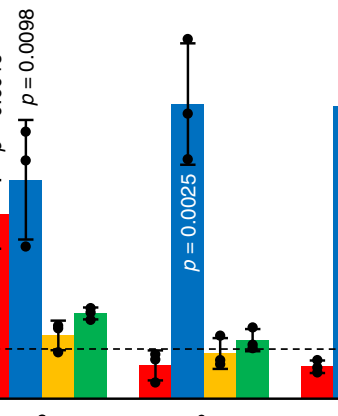

- GAPC1-OE

- GAPC2-OE

GAPC1mut-OE

- GAPC2mut-OE

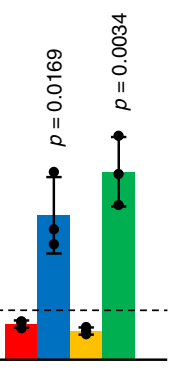

Fig. 7 Effects of GAPC mutation on heat response. a GAPC2 amino acid sequence. Colored are the lysine residues required for HsGAPDH nuclear localization (K121 and K231 in red, K255 in green) and found to be acetylated in E. coli (K130, K219, and K223 in blue, K255 in green). The lysines mutated in this study are underlined. $\mathbf{b}$ Immununoblotting of GAPC in nuclear fraction. Five-day-old transgenic seedlings overexpressing the indicated GAPC-Flag were untreated (Control) or treated at $40^{\circ} \mathrm{C}$ for $6 \mathrm{~h}$. Nuclei were isolated and GAPC was probed with an anti-Flag antibody by immunoblotting. Histone $\mathrm{H} 3$ was used as a nuclear marker. GAPCmut, GAPC with K121A/K231A. c Fluorescence images of Arabidopsis leaf cells. Five-day-old seedlings overexpressing the indicated GAPC-GFP were treated at $40^{\circ} \mathrm{C}$ for $6 \mathrm{~h}$ and observed under a confocal microscope. Arrows indicate the nucleus. Scale bars $=10 \mu \mathrm{m}$. $\mathbf{d}$ The number of cells with nuclear GAPC. Plants were treated and observed as in c. Cells with clear fluorescence in the nucleus were counted and shown here as $\%$ of total cells counted. Values are average \pm S.D. from 5 leaves independently treated. Black dots represent individual data points. e Quantitative measurement of heat response. Plants were untreated (Control) or treated at $40^{\circ} \mathrm{C}$ for $6 \mathrm{~h}$ and measured for seedling survival rate. Values are average \pm S.D. $P$ values indicate significant difference from WT determined by two-tailed student's $t$ test ( $n=3$ independent groups of $>30$ seedlings). Black dots represent individual data points. $\mathbf{f}$ Expression of heat-inducible genes. Total RNA was extracted from 5-day-old seedlings treated at $37^{\circ} \mathrm{C}$ for $5 \mathrm{~h}$ and quantitative RT-PCR was performed with gene-specific primers. Values are average \pm S.D. and shown as fold change to WT (dashed line). $P$ values indicate significant difference from WT determined by two-tailed student's $t$ test ( $n=3$ independent groups of $>10$ seedlings). Black dots represent individual data points.

translocation but neither acetylated in Arabidopsis nor required for OsGAPDH1 nuclear localization, are sufficient for the GAPC nuclear localization in response to heat. These signify the lysine acetylation requirement for stress-induced nuclear translocation conserved between animal and plant GAPDHs, but differential post-translational modifications for the GAPC subcellular distribution even within the plant kingdom. Recently, an E3 ubiquitin-protein ligase SEVEN IN ABSENTIA like 7 (SINAL7) was found to directly interact with GAPC1 in vitro, and nuclear GAPC abundance was decreased in sinal7 and increased by SINAL7 overexpression ${ }^{17,35}$. Interestingly, K231A mutation in GAPC1 abolished its interaction with SINAL7, and K76 in
GAPC1 corresponding to K74 in OsGAPDH1 was ubiquitinated by SINAL7 ${ }^{17}$. SINAL7 overexpression enhanced drought tolerance of Arabidopsis, increased DREB2A expression, and decreased stomatal aperture ${ }^{35}$. These data suggest a potential role of ubiquitination, particularly through $\mathrm{K} 231$, in heat-dependent nuclear translocation of GAPC. Taken together, different extracellular stimuli and their intracellular signaling messengers (e.g. heat, salt, osmolyte, heavy metal, reactive oxygen species, and lipid mediators) might differentially and specifically modify GAPC for nuclear access, possibly through cysteine sulfur-modification, lysine acetylation, ubiquitination, or yet unidentified post-translational modifications. 

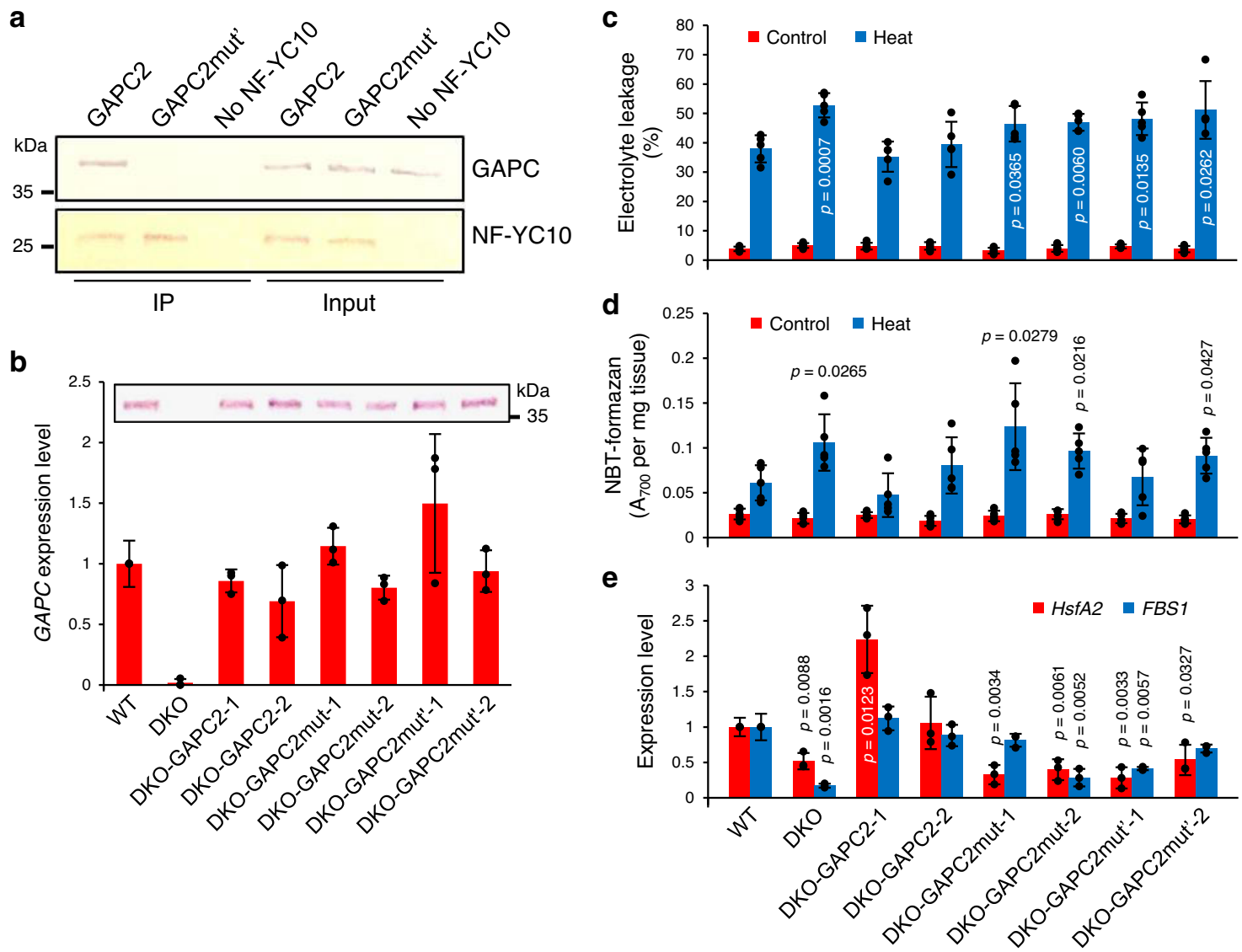

Fig. 8 Heat response of gapc1gapc2 (DKO) complemented with GAPC2 variants. a Co-immunoprecipitation of GAPC and NF-YC10. Purified GAPC2-6xHis or GAPC2mut'-6xHis was mixed with NF-YC10-6xHis-STREP. Immunoprecipitation (IP) was performed using an anti-STREP antibody, then immunoblotting was performed using an anti-6xHis antibody. Protein input is shown on the right. b GAPC expression levels. Total RNA was extracted from 10-day-old seedlings and quantitative RT-PCR was performed with GAPC2-specific primers. Values are average \pm S.D. from 3 independent seedlings and shown as fold change to WT. Black dots represent individual data points. DKO, double knockout mutant of GAPC. Inset, immunoblotting of GAPC. Total proteins were extracted from 5-dayold seedlings and GAPC was probed with the anti-GAPC antibody. c Electrolyte leakage. Conductivity was measured with true leaves from 2-week-old plants immediately after heat treatment at $40^{\circ} \mathrm{C}$ for $6 \mathrm{~h}$. Electrolyte leakage was calculated and shown here as $\%$ conductivity of total ions (boiled sample). Values are average \pm S.D. $P$ values indicate significantly higher than WT determined by two-tailed student's $t$ test ( $n=5$ leaves from independent plants). Black dots represent individual data points. d Reactive oxygen species production. Rosette leaves from 3-week-old plants were incubated with NBT immediately after heat treatment at $40^{\circ} \mathrm{C}$ for $6 \mathrm{~h}$. NBT-formazan was quantified as absorbance at $700 \mathrm{~nm}$ and shown here as per tissue weight. Values are average \pm S.D. $P$ values indicate significant difference from WT determined by two-tailed student's $t$ test ( $n=5$ leaves from independent plants). Black dots represent individual data points. e Expression of heat-inducible genes. Total RNA was extracted from 10-day-old seedlings treated at $37^{\circ} \mathrm{C}$ for $5 \mathrm{~h}$ and quantitative RT-PCR was performed with gene-specific primers. Values are average \pm S.D. and shown as fold change to WT. $P$ values indicate significantly lower than WT determined by two-tailed student's $t$ test ( $n=3$ independent seedlings). Black dots represent individual data points.

Another intriguing question is the metabolic implication of the nuclear translocation of GAPC under heat stress. Unlike animal GAPDH with only one cytosolic form, GAPDHs in higher plants are classified into three groups according to their subcellular locations: cytoplasmic GAPC for glycolysis, glycolytic GAPCp in nongreen plastids, and chloroplastic GAPA/B for photosynthetic $\mathrm{CO}_{2}$ fixation ${ }^{1,36-38}$. In addition, the Arabidopsis cytoplasm contains non-phosphorylating (NP)-GAPDH that catalyzes oxidation, instead of phosphorylation, of the substrate for cytoplasmic maintenance of $\mathrm{NADPH}^{39}$. Our previous analysis showed that the double knock of GAPC1 and GAPC2 resulted in significant changes in the level of glycolytic intermediates and the ratios of ATP/ADP and $\mathrm{NAD}(\mathrm{P}) \mathrm{H} / \mathrm{NAD}(\mathrm{P})^{28}$. However, the GAPC-deficient plants display no overt changes in vegetative growth, but decreased seed oil content compared to $\mathrm{WT}^{2,28}$. This complexity of the plant GAPDH families and tolerance of Arabidopsis plants to metabolic perturbation caused by GAPC alterations could be explained in part by the partial genetic redundancy of plant GAPDH. Under heat stress, only a small fraction of GAPC was translocated into nuclei based on our immunoblotting and microscopic analyses. Thus, the heat-induced nuclear translocation of GAPC might not drastically decrease the cytosolic glycolytic activity, and instead the major function of the stress-induced nuclear GAPCs is via regulation, including gene expression as shown in this study. However, heat stress may reduce the overall cytosolic glycolytic activity because GAPCs are potentially inactivated by the stress-induced oxidation. GAPDH, including GAPC, has been proposed to function as a redox sensor through its highly reactive cysteine for the regulation of energy metabolism ${ }^{5}$. In animal system, oxidative inactivation of GAPDH enables cells to redirect the carbohydrate metabolic flux from glycolysis to pentose phosphate pathway for NAPDH production $^{40,41}$.

Moonlighting of metabolic enzymes, such as GAPDH, provides a mechanistic link between metabolic activity and cellular regulation, and dysfunction of such regulation has severe physiological and pathological consequences in animal systems. Although the stress-induced nuclear moonlighting of GAPC has been described in many studies, downstream signaling target(s) and 
a
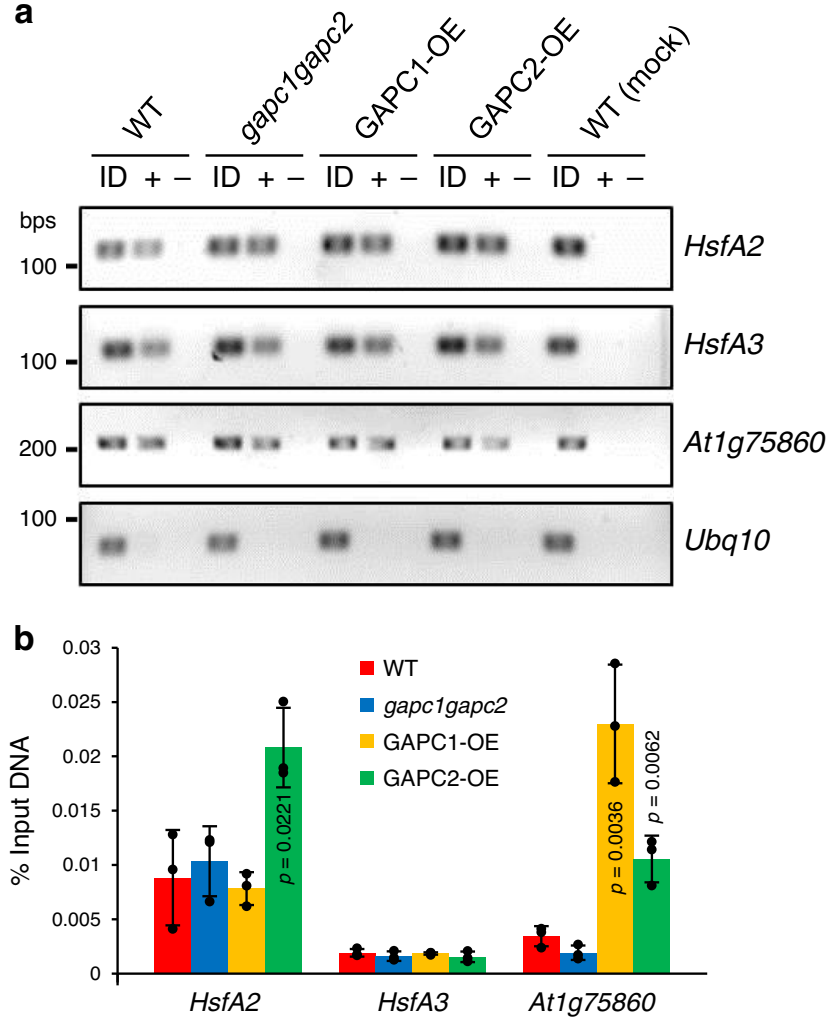

C

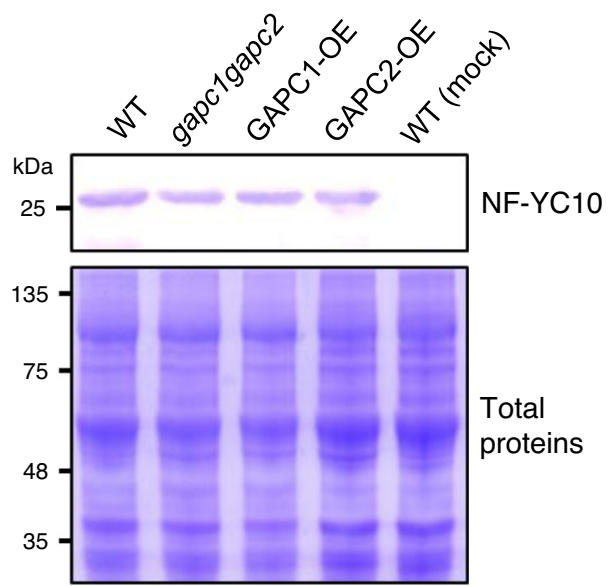

Fig. 9 Effect of GAPC on NF-YC10 binding to its target promoters. a PCR verification of the precipitated DNA. Chromatin immunoprecipitation (ChIP) was performed using anti-Flag antibody from heat-treated mesophyll protoplasts that were isolated from the GAPC-altered Arabidopsis lines indicated on the top and transfected with 35S:NF-YC10Flag. Input DNA (ID; see Methods for details) and DNA precipitated with $(+)$ or without $(-)$ the antibody were used for PCR with primers specific to the promoters of genes indicated on the right. Mock, non-transfected; Ubq10, ubiquitin10. b Quantification of the precipitated DNA. Quantitative PCR was performed with the DNA samples obtained from a. Values are average \pm S.D. and shown as \% of PCR product amplified from the input DNA. $P$ values indicate significant difference from WT determined by twotailed student's $t$ test ( $n=3$ independent pools of protoplasts). Black dots represent individual data points. c NF-YC10 protein abundance. After transfection, total proteins were extracted from the protoplasts and immunoblotting was performed with anti-Flag antibody (top). Coomassie blue staining is shown for loading control (bottom).

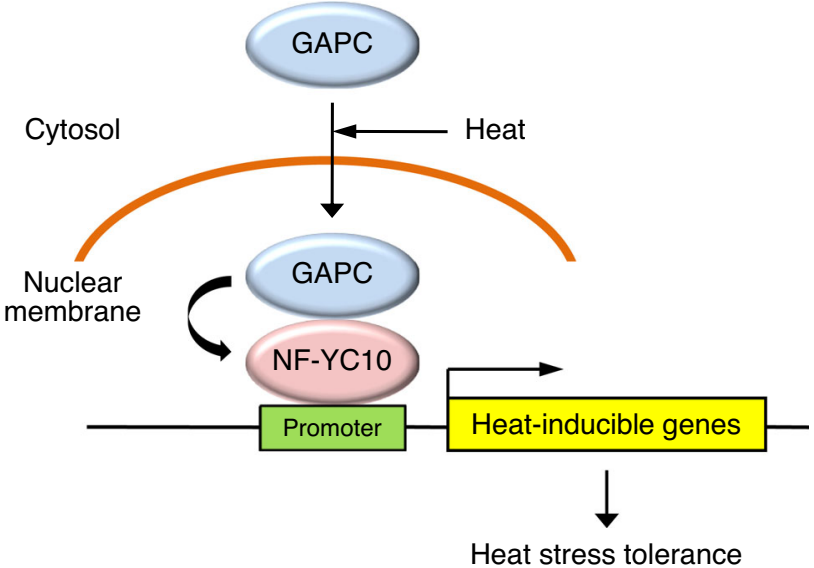

Fig. 10 Proposed model for GAPC-mediated heat response in

Arabidopsis. GAPC that normally exists in the cytosol enters the nucleus in response to heat. In the nucleus GAPC binds to the transcription factor NFYC10 to increase the expression of heat-inducible genes, rendering Arabidopsis tolerant to heat stress.

GAPC effects on its/their nuclear function have largely been unexplored. By demonstrating a heat-dependent action of GAPC in the nucleus, we show the apparent bifurcating property of plant GAPC that depends distinctively on catalytic activity for constitutive cellular process and on subcellular localization for stress response. Hence, our study brings significance in understanding the energy-efficient strategy that cells have developed to increase functional options without increasing the number of genes to avoid the highly energy-consuming processes, replication, transcription, and translation. Furthermore, our study could represent future directions to improve heat tolerance of crops by providing a molecular basis to adjust the balance between normal plant growth and stress response, as heat stress is an increasing problem for crop cultivation and food security.

\section{Methods}

Identification of GAPC-binding transcription factors. The transcription factor cDNA library was previously cloned and expressed in $E$. colit2. Briefly, a library containing 1498 individually cloned cDNA of Arabidopsis transcription factors ${ }^{24}$ was cloned into pDONR221 ${ }^{\mathrm{TM}}$ then $\mathrm{pET}$-53-DEST ${ }^{\mathrm{TM}}$ with $6 \mathrm{xHis}$ tag (Novagen) using Gateway ${ }^{\circledast}$ Recombination Kit (Life Technologies), and was transformed into Max Efficiency ${ }^{\circledR}$ DH5a Competent Cells (Life Technologies) for plasmid propagation then into E. coli Rosetta $^{\mathrm{TM}}$ (DE3) for protein expression. Sequence of primers used for cloning is provided in Supplementary Table 1. Based on estimate by Clarke and Carbon $(1976)^{43}$, we calculated the number of colonies required to cover 1498 different clones as $N=\ln (1-P) / \ln (1-F)$, where $P=$ desired probability of finding the clone of interest (typically 0.99 ) and $F=$ fractional portion of the library present in a single clone (1/1498). $N$ was estimated at a maximum of 6900 , so we used over 7000 colonies for protein extraction. Colonies were pooled and incubated in Luria-Bertani (LB) media at $15^{\circ} \mathrm{C}$ for $6 \mathrm{~h}$ for induction. Proteins were purified using Ninitrilotriacetic acid (NTA) Agarose (QIAGEN). Fifty $\mu \mathrm{g}$ of the purified proteins were co-immunoprecipitated as described below with GAPC2-Flag isolated from Arabidopsis overexpressing the recombinant protein or from control plants with empty vector (EV) using an anti-Flag antibody (GenScript A00187; 1:100 diluted). The GAPC2-bound proteins were resolved by SDS-PAGE and visualized by Coomassie blue. Each whole lane containing protein bands was carefully excised from the gel and divided into several pieces, and the proteins were in-gel digested with trypsin (SigmaAldrich) at $37^{\circ} \mathrm{C}$ overnight, according to the manufacturer's instructions. The digested peptides were run on the LC-tandem MS using an Orbitrap Fusion Lumos (Thermo Scientific) coupled with a Dionex RSLCnano HPLC (Thermo Scientific). The samples were dried down to concentrate and resuspended in $10 \mu \mathrm{L}$ of $5 \%$ $(\mathrm{v} / \mathrm{v})$ acetonitrile/0.1\% (v/v) formic acid mixture. $5 \mu \mathrm{L}$ was injected for LC-MS/MS on 2-h gradient separation and data acquisition. Peptides were resolved using $75 \mu \mathrm{m} \times$ $50 \mathrm{~cm}$ PepMap C18 column (Thermo Scientific). The database search was performed with peptide mass fingerprint data using Mascot (v2.5.1.0) database search engine (Matrix Science) against the Arabidopsis thaliana proteome database (Uniprot.org). Mascot was searched with a fragment ion mass tolerance of $0.60 \mathrm{Da}$ and a parent ion tolerance of $10 \mathrm{ppm}$. The criteria for a significant protein identification were both at least two unique peptides per protein identified and each peptide showing a 
probability $>99 \%$. Data are available via ProteomeXchange with identifier PXD018945 [https://doi.org/10.6019/PXD018945]. Proteins found from GAPC2 but not from EV (designated as ' $\mathrm{S}$ ' and ' $\mathrm{C}$ ' in the data repository, respectively) were considered as GAPC-binding candidates.

Plant materials and growth condition. All Arabidopsis plants used were Columbia-0 ecotype of Arabidopsis thaliana. T-DNA insertional knockout mutants were obtained from the Arabidopsis Biological Resource Center (Ohio State University) and homozygotes were confirmed by PCR-based genotyping. All GAPC transgenic plants and GAPC double knockout mutants (gapclgapc2) were previously generated, and the floral dipping method was applied to generate all other transgenic plants used in this study ${ }^{2,28}$. Seeds were surface-sterilized with $70 \%(\mathrm{v} / \mathrm{v})$ ethanol, and then $20 \%(\mathrm{v} / \mathrm{v})$ bleach, followed by washing 5 times with water. Sterilized seeds were stratified at $4{ }^{\circ} \mathrm{C}$ for 2 days prior to germination. Plants were germinated and grown on $1 / 2$ strength of Murashige and Skoog (MS) plates with $1 \%$ (w/v) sucrose and $0.8 \%$ $(\mathrm{w} / \mathrm{v})$ agar at $22^{\circ} \mathrm{C}$ under light cycles of 16 -h light/8-h dark.

Immunoprecipitation. Immunoprecipitation was performed using an anti-Flag antibody (GenScript A00187; 1:100 diluted) or anti-STREP antibody (GenScript A00626; 1:100 diluted) and Protein A-agarose (Sigma-Aldrich), according to the manufacturer's instructions. GAPCs were Flag-tagged whereas NF-YC10 was STREPtagged. Plant tissues were ground with liquid nitrogen and incubated in protein extraction buffer ( $50 \mathrm{mM}$ Tris-HCl pH7.3, $50 \mathrm{mM} \mathrm{NaCl}, 5 \%$ (v/v) glycerol, $1 \mathrm{mM}$ DTT) containing a protease inhibitor cocktail (Sigma-Aldrich) on ice for $30 \mathrm{~min}$. Supernatant after centrifugation at $12000 \times \mathrm{g}$ for $10 \mathrm{~min}$ at $4{ }^{\circ} \mathrm{C}$ was used as a protein extract. $10 \mathrm{mg}$ of Protein A-agarose was swollen with $0.2 \mathrm{~mL}$ of buffer A $(20 \mathrm{mM}$ $\mathrm{NaH}_{2} \mathrm{PO}_{4} \mathrm{pH} 8.0,150 \mathrm{mM} \mathrm{NaCl}$ ) for $1 \mathrm{~h}$ and washed with buffer $\mathrm{A}$. The protein extract was mixed with $5 \mu \mathrm{g}$ of antibody, the swollen Protein A-agarose, and buffer A to the final volume of $1 \mathrm{~mL}$, and gently agitated overnight at $4{ }^{\circ} \mathrm{C}$. The mixture was washed five times with buffer A by centrifugation at $2500 \times g$ for $1 \mathrm{~min}$. Target proteins were added to the immunocomplex and gently agitated overnight at $4{ }^{\circ} \mathrm{C}$. The mixture was washed five times with buffer A by centrifugation at $2500 \times g$ for $1 \mathrm{~min}$. SDS-PAGE loading buffer was added and boiled for $10 \mathrm{~min}$. After brief centrifugation, the supernatant was used for SDS-PAGE or immunoblotting.

Immunoblotting. Protein samples were dissolved in SDS-PAGE loading buffer, boiled for $5 \mathrm{~min}$, and loaded on $10 \%(\mathrm{v} / \mathrm{v})$ polyacrylamide gel. After running the gel at $100 \mathrm{~V}$ for $\sim 1 \mathrm{~h}$, proteins were electrophoretically transferred onto a polyvinylidene fluoride (PVDF) membrane using Semidry Trans-Blot apparatus (Bio$\mathrm{Rad})$ at $20 \mathrm{~V}$ for $20 \mathrm{~min}$. The membrane was blocked in TBST buffer containing $5 \%$ $(\mathrm{w} / \mathrm{v})$ nonfat milk for $1 \mathrm{~h}$, followed by washing three times with TBST buffer. The membrane was incubated for $1 \mathrm{~h}$ with primary antibodies: anti-Flag (GenScript A00187; 1:2000 diluted), anti-6xHis (GenScript A00186; 1:2000 diluted), antiHistone H3 (GenScript A01502; 1:1000 diluted), anti-PEPC (Rockland 100-4163; 1:500 diluted), and anti-GAPC (Agrisera AS15-2894; 1:2000 diluted). After washing three times with TBST buffer, the membrane was incubated with secondary antibodies (Sigma-Aldrich) from mouse or rabbit conjugated with alkaline phosphatase for $1 \mathrm{~h}$. Proteins were visualized by alkaline phosphatase conjugate substrate (Bio-Rad) according to the manufacturer's instructions.

Bimolecular fluorescence complementation (BiFC). The BiFC vectors were constructed, described, and provided by Walter et al. $(2004)^{44}$. GAPC1 or GAPC2 cDNA was cloned into pSPYNE vector (GAPC-YFP $)$, and NF-YC10 cDNA was cloned into pSPYCE vector (NF-YC10-YFPC). Sequence of primers used for cloning is provided in Supplementary Table 1. The constructs were transformed into $\mathrm{C} 58 \mathrm{C} 1$ Agrobacterium tumefaciens strain and grown to stationary phase. Bacterial cells were collected and resuspended in solution containing $10 \mathrm{mM}$ MES, pH 5.7, $10 \mathrm{mM} \mathrm{MgCl}_{2}$, and $150 \mathrm{mg} \mathrm{ml}^{-1}$ acetosyringone. Three-week-old Nicotiana benthamiana leaves were infiltrated with the mixed bacteria $\left(\mathrm{GAPC}-\mathrm{YFP}^{\mathrm{N}}\right.$ and NF-YC10-YFPC) solutions. YFP fluorescence was examined in tobacco leaves using a Zeiss LSM 510 confocal microscope, with a $488-\mathrm{nm}$ excitation mirror and a 505to 530-nm filter to record images. GPA1-YFP ${ }^{\mathrm{N}}$ and PLDa1-YFPC were used as negative controls for NF-YC10-YFPC and GAPC-YFPN , respectively and as a positive control with each other. For quantification of BiFC, confocal images from randomly chosen regions of infiltrated tobacco leaves were obtained with the same settings (magnification, focal plane, area, etc). Background-subtracted fluorescence intensity was measured by ImageJ software (v1.52a).

Quantitative real-time PCR (qRT-PCR). Total RNA was extracted from plant tissues using TRIzol Reagent (Life Technologies) per the manufacturer's instructions. RNA was quantified by Nanodrop 2000 spectrophotometer (Thermo Scientific) and checked for integrity by agarose gel electrophoresis. cDNA was synthesized by SuperScript ${ }^{\circledast}$ III reverse transcriptase (Life Technologies) with $1 \mu \mathrm{g}$ of RNA and $0.5 \mu \mathrm{g}$ of oligo $(\mathrm{dT})_{18}$ primers, according to the manufacturer's instructions. The reaction was at $50^{\circ} \mathrm{C}$ for $30 \mathrm{~min}$ with preincubation at $65^{\circ} \mathrm{C}$ for $5 \mathrm{~min}$ and enzyme inactivation at $70^{\circ} \mathrm{C}$ for $15 \mathrm{~min}$. The cDNA was amplified with a Taq polymerase using gene-specific primers through the following thermal cycling conditions: preincubation at $95^{\circ} \mathrm{C}$ for $2 \mathrm{~min}, 40$ cycles of $95^{\circ} \mathrm{C}$ for $30 \mathrm{~s}, 55^{\circ} \mathrm{C}$ for $30 \mathrm{~s}$ and $68^{\circ} \mathrm{C}$ for $1 \mathrm{~min}$, and final extension at $68^{\circ} \mathrm{C}$ for $5 \mathrm{~min}$. The PCR progress was monitored by adding SYBR
Green dye using StepOnePlus ${ }^{\mathrm{TM}}$ Real-Time PCR System (Applied Biosystems), and data were processed by StepOne ${ }^{\mathrm{TM}}$ Software (v2.0.2). The gene expression was normalized with ubiquitin 10 as an internal standard. Sequence of primers used for qRT-PCR is provided in Supplementary Table 1.

Chlorophyll content measurement. Leaves cut from seedling samples were weighed $(W)$, added to $2 \mathrm{~mL}$ of $95 \%(\mathrm{v} / \mathrm{v})$ ethanol in an airtight tube, and agitated at room temperature in dark overnight until leaves turned to white. After brief centrifugation, the ethanol phase was taken and measured with a spectrophotometer at wave lengths of $665 \mathrm{~nm}\left(A_{665}\right), 649 \mathrm{~nm}\left(A_{649}\right)$, and $470 \mathrm{~nm}\left(A_{470}\right)$. The amount of chlorophyll a (Ca) was calculated as $\mathrm{Ca}=13.95 \times A_{665}-6.88 \times A_{649}$ chlorophyll b (Cb) as $\mathrm{Cb}=24.96 \times A_{649}-7.32 \times A_{665}$; and carotenoids $(\mathrm{Cc})$ as $\mathrm{Cc}$ $=\left(1000 \times A_{470}-2.05 \times \mathrm{Ca}-114.8 \times \mathrm{Cb}\right) / 245$. The total content of chlorophyll per fresh weight was calculated as $\mathrm{C}=2 \times(\mathrm{Ca}+\mathrm{Cb}+\mathrm{Cc}) / W$.

Electrolyte leakage and reactive oxygen species measurement. Rosette leaves were excised from 3-week-old plants grown on soil immediately after heat treatment and incubated in $15 \mathrm{~mL}$ ultrapure (Milli-Q) water for $1 \mathrm{~h}$ at room temperature. Conductivity was measured using Mettler Toledo MC-126 conductivity meter. Samples were then boiled for $15 \mathrm{~min}$ and measured again after cooling down to room temperature. Electrolyte leakage was calculated as \% conductivity of total ions from the boiled sample. Superoxide anion $\left(\mathrm{O}_{2}{ }^{-}\right)$was measured by staining with nitrotetrazolium blue chloride (NBT). Rosette leaves were cut off from 3week-old plants after heat treatment and vacuum-infiltrated with $0.1 \%$ (w/v) NBT dissolved in $50 \mathrm{mM}$ potassium phosphate and $10 \mathrm{mM}$ sodium azide. The tissues were incubated in the NBT solution for $1 \mathrm{~h}$ in the dark with gentle agitation and washed with $95 \%(\mathrm{v} / \mathrm{v})$ ethanol until completely bleached. Formazan formed was extracted with a mixture of $2 \mathrm{M}$ potassium hydroxide and chloroform (1:1, v/v), dried under gentle stream of nitrogen gas, and dissolved in a mixture of DMSO and $2 \mathrm{M}$ potassium hydroxide $(1: 1, \mathrm{v} / \mathrm{v})$. Absorbance of the final solution was measured at $700 \mathrm{~nm}$ using a spectrophotometer.

Nuclei isolation. Approximately $1 \mathrm{~g}$ of plant tissue was ground with liquid nitrogen in mortar and pestle and mixed with $5 \mathrm{~mL}$ of buffer A $(10 \mathrm{mM}$ Tris- $\mathrm{HCl}$ pH7.6, $0.5 \mathrm{M}$ sucrose, $1 \mathrm{mM}$ spermidine, $4 \mathrm{mM}$ spermine, $10 \mathrm{mM}$ EDTA, and 80 $\mathrm{mM} \mathrm{KCl}$. The tissue homogenate was filtered through 4 layers of Miracloth and centrifuged at $3000 \times g$ for $5 \mathrm{~min}$. Supernatant was centrifuged at $16000 \times g$ for $30 \mathrm{~min}$ and used as cytosolic fraction, and pellet (nuclear suspension from the $3000 \times g$ centrifugation) was gently resuspended in $1 \mathrm{~mL}$ of buffer B $(50 \mathrm{mM}$ Tris$\mathrm{HCl} \mathrm{pH7.8,} 5 \mathrm{mM} \mathrm{MgCl}$, $10 \mathrm{mM} \beta$-mercaptoethanol, and 20\% (v/v) glycerol). Discontinuous Percoll ${ }^{\mathrm{TM}}$ (Amersham Biosciences) gradient was prepared with $2 \mathrm{~mL}$ each of $40 \%(\mathrm{v} / \mathrm{v}), 60 \%$, and $80 \%$ (top to bottom) Percoll ${ }^{\mathrm{TM}}$ dissolved in buffer C (25 mM Tris-HCl pH7.5, $0.44 \mathrm{M}$ sucrose, and $\left.10 \mathrm{mM} \mathrm{MgCl}_{2}\right)$ on $2 \mathrm{~mL}$ of $2 \mathrm{M}$ sucrose cushion. The nuclear suspension was gently loaded on top of the Percoll ${ }^{\mathrm{TM}}$ gradient and centrifuged at $4000 \times g$ for $30 \mathrm{~min}$. Nuclear layer (light green) just above the $2 \mathrm{M}$ sucrose cushion was carefully taken and washed twice with $1 \mathrm{~mL}$ of buffer $\mathrm{A}$ at $6000 \times g$ for $5 \mathrm{~min}$. The nuclear pellet was gently resuspended in $0.2 \mathrm{~mL}$ of buffer $\mathrm{B}$ and used for further experiments.

GAPC activity assay. Catalytic activity of GAPC was determined spectrophotometically by measuring the concomitant reduction of $\mathrm{NAD}^{+}$in a two-step reaction: production of glyceraldehyde-3-phosphate from fructose-1,6-bisphosphate by aldolase followed by 1,3-bisphosphoglycerate from glyceraldehyde-3-phosphate by GAPC. Reaction mixture contained $50 \mathrm{mM}$ triethanolamine- $\mathrm{HCl}$, pH8.5, 4 mM NAD ,$+ 1.2 \mathrm{mM}$ fructose-1,6-bisphosphate, $10 \mathrm{mM}$ sodium arsenate, 1 unit of aldolase from rabbit muscle, and 5 20 $\mu \mathrm{g}$ of purified GAPC or total protein extract from Arabidopsis in a final volume of $1 \mathrm{~mL}$. Reaction was initiated by the addition of fructose1,6-bisphosphate at room temperature for $30 \mathrm{~min}$, then immediately measured for the NADH formation at $340 \mathrm{~nm}$ using a spectrophotometer.

Site-directed mutagenesis and GAPC-complemented gapc1gapc2. PCR-based lysine mutation of GAPC was carried out using QuikChange Multi Site-Directed Mutagenesis Kit (Agilent Technologies, Santa Clara, CA) according to the manufacturer's instruction. In brief, GAPC cDNA previously cloned in pET-28a(+) vector ${ }^{7}$ was used as a template. Sequence of the mutagenic primers with lysine (AAA or AAG) substituted to alanine (GCA or GCG) is provided in Supplementary Table 1 Mutant strand synthesis was performed by PCR at $95^{\circ} \mathrm{C}$ for 1 min followed by 30 cycles of $95^{\circ} \mathrm{C} 1 \mathrm{~min}, 55^{\circ} \mathrm{C} 1 \mathrm{~min}$, and $65^{\circ} \mathrm{C} 10 \mathrm{~min}$. The PCR product was incubated with restriction enzyme $D p n$ I at $37^{\circ} \mathrm{C}$ for $1 \mathrm{~h}$ to digest template and used for transformation of XL10-Gold Ultracompetent Cells. The correct mutations were verified by DNA sequencing on both strands. The lysine-mutated GAPC cDNA was subcloned into p35S-FAST/eYFP vector for plant expression ${ }^{7}$. In the resulting DNA constructs p35S-FAST/eYFP-GAPCmut and p35S-FAST/eYFP-GAPCmut', the region containing CaMV-35S promoter and eYFP was replaced with the GAPC promoter sequence ( $-633 \sim-1$ from the start codon of GAPC2) by cloning at EcoRI and BamHI sites of the vector. The DNA constructs pFAST-GAPC::GAPCmut and pFAST-GAPC::GAPCmut' were then transformed into Agrobacteria, and then into gapclgapc2 by floral dipping for the generation of GAPCmut- or GAPCmut'- 
complemented gapclgapc2. Transgenic plants were selected on kanamycin and further PCR-confirmed with genomic DNA for the presence of the transgene.

Protoplast isolation and transfection. Mesophyll protoplasts were isolated from 4-week-old Arabidopsis leaves and transfected with pFAST-35S::NF-YC10-Flag ${ }^{45}$. Briefly, $\sim 0.1 \mathrm{~g}$ of leaf strips $(\sim 1 \mathrm{~mm}$ in width) were vacuum-infiltrated with $5 \mathrm{~mL}$ enzyme solution (20 mM MES pH 5.7, 1.5\% (w/v) cellulose R10, 0.4\% (w/v) macerozyme R10, $0.4 \mathrm{M}$ mannitol, $20 \mathrm{mM} \mathrm{KCl}, 10 \mathrm{mM} \mathrm{CaCl}_{2}, 0.1 \%$ (w/v) BSA) and incubated for $6 \mathrm{~h}$ in the dark for cell wall digestion. The protoplast suspension diluted with equal volume of W5 buffer (2 mM MES pH 5.7, $154 \mathrm{mM} \mathrm{NaCl}, 125$ $\mathrm{mM} \mathrm{CaCl}, 5 \mathrm{mM} \mathrm{KCl}$ ) was filtered through a nylon mesh to remove undigested tissues, then centrifuged at $200 \times g$ for $2 \mathrm{~min}$. Pellet was washed with $1 \mathrm{~mL}$ W5 solution at $200 \times g$ for $2 \mathrm{~min}$ and re-suspended in $1 \mathrm{~mL}$ MMG buffer $(4 \mathrm{mM}$ MES pH 5.7, $0.4 \mathrm{M}$ mannitol, $15 \mathrm{mM} \mathrm{MgCl} 2$ ). $100 \mu \mathrm{L}$ protoplast suspension was mixed with $10 \mu \mathrm{g}$ DNA and $110 \mu \mathrm{L}$ PEG solution $(20 \%$ (w/v) PEG4000, $0.2 \mathrm{M}$ mannitol, $100 \mathrm{mM} \mathrm{CaCl}_{2}$ ) for $1 \mathrm{~h}$ for transfection. The sample diluted with equal volume of W5 buffer was centrifuged at $200 \times g$ for $2 \mathrm{~min}$. Pellet was re-suspended in $1 \mathrm{~mL}$ WI buffer (4 mM MES pH 5.7, $0.5 \mathrm{M}$ mannitol, $20 \mathrm{mM} \mathrm{KCl}$ ).

Chromatin immunoprecipitation (ChIP). ChIP was performed according to an existing method ${ }^{46}$. For crosslinking, protoplasts washed with PBS buffer (pH 7.4) at $1500 \times g$ for $2 \mathrm{~min}$ were incubated in $1 \mathrm{~mL}$ PBS containing $1 \%(\mathrm{v} / \mathrm{v})$ formaldehyde for $10 \mathrm{~min}$, then $0.1 \mathrm{M}$ glycine was added for $5 \mathrm{~min}$ to quench crosslinking. Protoplasts washed twice with ice-cold PBS were incubated with $1 \mathrm{~mL}$ harvest buffer (10 mM DTT, $100 \mathrm{mM}$ Tris-HCl pH 9.4) for $15 \mathrm{~min}$. For chromatin extraction, protoplasts were washed stepwise with ice-cold PBS, nuclei wash buffer with Triton X-100 ( $0.25 \%(\mathrm{v} / \mathrm{v})$ Triton X-100, $10 \mathrm{mM}$ EDTA, $0.5 \mathrm{mM}$ EGTA, $10 \mathrm{mM}$ HEPES pH 6.5), and nuclei wash buffer without Triton X-100 (200 mM NaCl, $1 \mathrm{mM}$ EDTA, $0.5 \mathrm{mM}$ EGTA, $10 \mathrm{mM}$ HEPES $\mathrm{pH}$ 6.5), then re-suspended in $300 \mu \mathrm{L}$ icecold nuclei lysis buffer (1\% (w/v) SDS, $10 \mathrm{mM}$ EDTA, $50 \mathrm{mM}$ Tris-HCl pH 8.0, $1 \mathrm{X}$ protease inhibitor cocktail). DNA was sheared by sonication for $1 \mathrm{~min}$ and centrifuged at $10000 \times g$ for $5 \mathrm{~min}$. An aliquot of supernatant was reserved as 'input DNA'. $1 \mu \mathrm{g}$ of an anti-Flag antibody (GenScript A00187) was added and incubated overnight at $4^{\circ} \mathrm{C}$, then $100 \mu \mathrm{L}$ swollen protein A agarose (Sigma-Aldrich) was added and incubated for $1 \mathrm{~h}$ at $4{ }^{\circ} \mathrm{C}$. The sample was washed stepwise with low salt wash buffer $(0.1 \%(\mathrm{w} / \mathrm{v})$ SDS, $1 \%$ (v/v) Triton X-100, 2 mM EDTA, $20 \mathrm{mM}$ Tris$\mathrm{HCl} \mathrm{pH} \mathrm{8.0,150} \mathrm{mM} \mathrm{NaCl),} \mathrm{high} \mathrm{salt} \mathrm{wash} \mathrm{buffer} \mathrm{(low} \mathrm{salt} \mathrm{buffer} \mathrm{with} 500 \mathrm{mM}$ $\mathrm{NaCl})$, LiCl wash buffer $(0.25 \mathrm{M} \mathrm{LiCl}, 1 \%$ (v/v) NP-40, 1 mM EDTA, $10 \mathrm{mM}$ Tris$\mathrm{HCl} \mathrm{pH} 8.0,1 \%(\mathrm{w} / \mathrm{v})$ sodium deoxycholate), and twice with TE buffer $(10 \mathrm{mM}$ Tris-HCl pH 8.0, 1 mM EDTA). $150 \mu \mathrm{L}$ elution buffer (1\% (w/v) SDS, $0.1 \mathrm{M}$ sodium bicarbonate) was added and incubated at $65^{\circ} \mathrm{C}$ for $15 \mathrm{~min}$. The sample was centrifuged at $5000 \times g$ for $3 \mathrm{~min}$ and supernatant was incubated with $0.2 \mathrm{M} \mathrm{NaCl}$ at $65^{\circ} \mathrm{C}$ for $6 \mathrm{~h}$ to reverse crosslinking, then incubated with $1 \mu \mathrm{g}$ proteinase $\mathrm{K}$ at 37 ${ }^{\circ} \mathrm{C}$ for $1 \mathrm{~h}$. DNA was purified using Gel/PCR DNA Fragments Extraction Kit (IBI Scientific) according to manufacturer's instruction.

Reporting summary. Further information on research design is available in the Nature Research Reporting Summary linked to this article.

\section{Data availability}

The data that support the findings of this study are available within the paper and its supplementary information, or are available from the corresponding author upon reasonable request. Source data are provided with this paper. The mass spectrometry proteomics data have been deposited to the ProteomeXchange Consortium via the PRIDE $^{47}$ partner repository with the dataset identifier PXD018945 [https://doi.org/ 10.6019/PXD018945].

Received: 6 May 2019; Accepted: 23 June 2020;

Published online: 10 July 2020

\section{References}

1. Rius, S. P., Casati, P., Iglesias, A. A. \& Gomez-Casati, D. F. Characterization of Arabidopsis lines deficient in GAPC-1, a cytosolic NAD-dependent glyceraldehyde-3-phosphate dehydrogenase. Plant Physiol. 148, 1655-1667 (2008)

2. Guo, L. et al. Cytosolic glyceraldehyde-3-phosphate dehydrogenases interact with phospholipase $\mathrm{D} \delta$ to transduce hydrogen peroxide signals in the Arabidopsis response to stress. Plant Cell 24, 2200-2212 (2012)

3. Zaffagnini, M., Fermani, S., Costa, A., Lemaire, S. D. \& Trost, P. Plant cytoplasmic GAPDH: redox post-translational modifications and moonlighting properties. Front. Plant Sci. 4, 450 (2013).

4. Hildebrandt, T., Knuesting, J., Berndt, C., Morgan, B. \& Scheibe, R. Cytosolic thiol switches regulating basic cellular functions: GAPDH as an information hub? Biol. Chem. 396, 523-537 (2015).
5. Schneider, M., Knuesting, J., Birkholz, O., Heinisch, J. J. \& Scheibe, R. Cytosolic GAPDH as a redox-dependent regulator of energy metabolism. BMC Plant Biol. 18, 184 (2018).

6. Vescovi, M. et al. Nuclear accumulation of cytosolic glyceraldehyde-3phosphate dehydrogenase in cadmium-stressed Arabidopsis roots. Plant Physiol. 162, 333-346 (2013).

7. Kim, S.-C., Guo, L. \& Wang, X. Phosphatidic acid binds to cytosolic glyceraldehyde-3-phosphate dehydrogenase and promotes its cleavage in Arabidopsis. J. Biol. Chem. 288, 11834-11844 (2013).

8. Henry, E., Fung, N., Liu, J., Drakakaki, G. \& Coaker, G. Beyond glycolysis: GAPDHs are multi-functional enzymes involved in regulation of ROS, autophagy, and plant immune responses. PLoS Genet. 11, e1005199 (2015).

9. Liu, T. et al. Cytosolic glyceraldehyde-3-phosphate dehydrogenases play crucial roles in controlling cold-induced sweetening and apical dominance of potato (Solanum tuberosum L.) tubers. Plant Cell Environ. 40, 3043-3054 (2017).

10. Ruiz-Ruiz, S. et al. Citrus tristeza virus co-opts glyceraldehyde 3-phosphate dehydrogenase for its infectious cycle by interacting with the viral-encoded protein p23. Plant Mol. Biol. 98, 363-p373 (2018).

11. Han, S. et al. Cytoplastic glyceraldehyde-3-phosphate dehydrogenases interact with ATG3 to negatively regulate autophagy and immunity in Nicotiana benthamiana. Plant Cell 27, 1316-1331 (2015).

12. Kaido, M. et al. GAPDH-A recruits a plant virus movement protein to cortical virus replication complexes to facilitate viral cell-to-cell movement. PLoS Pathog. 10, e1004505 (2014).

13. Holtgrefe, S. et al. Regulation of plant cytosolic glyceraldehyde 3-phosphate dehydrogenase isoforms by thiol modifications. Physiol. Plant. 133, 211-228 (2008)

14. Aroca, A., Schneider, M., Scheibe, R., Gotor, C. \& Romero, L. C. Hydrogen sulfide regulates the cytosolic/nuclear partitioning of glyceraldehyde-3phosphate dehydrogenase by enhancing its nuclear localization. Plant Cell Physiol. 58, 983-992 (2017).

15. Testard, A. et al. Calcium-and nitric oxide-dependent nuclear accumulation of cytosolic glyceraldehyde-3-phosphate dehydrogenase in response to long chain bases in tobacco BY-2 cells. Plant Cell Physiol. 57, 2221-2231 (2016).

16. Zhang, H., Zhao, Y. \& Zhou, D. X. Rice NAD ${ }^{+}$-dependent histone deacetylase OsSRT1 represses glycolysis and regulates the moonlighting function of GAPDH as a transcriptional activator of glycolytic genes. Nucleic Acids Res. 45, 12241-12255 (2017)

17. Peralta, D. A., Araya, A., Busi, M. V. \& Gomez-Casati, D. F. The E3 ubiquitin-ligase SEVEN IN ABSENTIA like 7 mono-ubiquitinates glyceraldehyde-3-phosphate dehydrogenase 1 isoform in vitro and is required for its nuclear localization in Arabidopsis thaliana. Int. J. Biochem. Cell Biol. 70, 48-56 (2016).

18. Sirover, M. A. New nuclear functions of the glycolytic protein, glyceraldehyde-3phosphate dehydrogenase, in mammalian cells. J. Cell. Biochem. 95, 45-52 (2005).

19. Boukouris, A. E., Zervopoulos, S. D. \& Michelakis, E. D. Metabolic enzymes moonlighting in the nucleus: metabolic regulation of gene transcription. Trends Biochem. Sci. 41, 712-730 (2016).

20. Chuang, D. M., Hough, C. \& Senatorov, V. V. Glyceraldehyde-3-phosphate dehydrogenase, apoptosis, and neurodegenerative diseases. Annu. Rev. Pharmacol. Toxicol. 45, 269-290 (2005).

21. Colell, A., Green, D. R. \& Ricci, J. E. Novel roles for GAPDH in cell death and carcinogenesis. Cell Death Differ. 16, 1573-1581 (2009).

22. Butterfield, D. A., Hardas, S. S. \& Lange, M. L. B. Oxidatively modified glyceraldehyde-3-phosphate dehydrogenase (GAPDH) and Alzheimer's disease: many pathways to neurodegeneration. J. Alzheimer's Dis. 20, 369-393 (2010).

23. Guo, C., Liu, S. \& Sun, M. Z. Novel insight into the role of GAPDH playing in tumor. Clin. Transl. Oncol. 15, 167-172 (2013).

24. Mitsuda, N. et al. Efficient yeast one-/two-hybrid screening using a library composed only of transcription factors in Arabidopsis thaliana. Plant Cell Physiol. 51, 2145-2151 (2010).

25. Zhao, J. \& Wang, X. Arabidopsis phospholipase Da1 interacts with the heterotrimeric G-protein $\alpha$-subunit through a motif analogous to the DRY motif in G-protein-coupled receptors. J. Biol. Chem. 279, 1794-1800 (2004).

26. Roy Choudhury, S. \& Pandey, S. The role of PLDa1 in providing specificity to signal-response coupling by heterotrimeric G-protein components in Arabidopsis. Plant J. 86, 50-61 (2016).

27. Sato, H. et al. Arabidopsis DPB3-1, a DREB2A interactor, specifically enhances heat stress-induced gene expression by forming a heat stressspecific transcriptional complex with NF-Y subunits. Plant Cell 26, 4954-4973 (2014).

28. Guo, L. et al. Cytosolic phosphorylating glyceraldehyde-3-phosphate dehydrogenases affect Arabidopsis cellular metabolism and promote seed oil accumulation. Plant Cell 26, 3023-3035 (2014).

29. Schmid, M. et al. A gene expression map of Arabidopsis thaliana development. Nat. Genet. 37, 501-506 (2005). 
30. Ventura, M. et al. Nuclear translocation of glyceraldehyde-3-phosphate dehydrogenase is regulated by acetylation. Int. J. Biochem. Cell Biol. 42, 1672-1680 (2010).

31. Finkemeier, I., Laxa, M., Miguet, L., Howden, A. J. \& Sweetlove, L. J. Proteins of diverse function and subcellular location are lysine acetylated in Arabidopsis. Plant Physiol. 155, 1779-1790 (2011).

32. Zhao, H. et al. The Arabidopsis thaliana nuclear factor $\mathrm{Y}$ transcription factors. Front. Plant Sci. 7, 2045 (2017).

33. Wawer, I. et al. Regulation of Nicotiana tabacum osmotic stress-activated protein kinase and its cellular partner GAPDH by nitric oxide in response to salinity. Biochem. J. 429, 73-83 (2010).

34. Hartl, M. et al. Lysine acetylome profiling uncovers novel histone deacetylase substrate proteins in Arabidopsis. Mol. Syst. Biol. 13, 949 (2017).

35. Peralta, D. A., Araya, A., Gomez-Casati, D. F. \& Busi, M. V. Over-expression of SINAL7 increases biomass and drought tolerance, and also delays senescence in Arabidopsis. J. Biotechnol. 283, 11-21 (2018).

36. Cerff, R. \& Chambers, S. E. Subunit structure of higher plant glyceraldehyde3-phosphate dehydrogenases (EC 1.2. 1.12 and EC 1.2. 1.13). J. Biol. Chem. 254, 6094-6098 (1979).

37. Petersen, J., Brinkmann, H. \& Cerff, R. Origin, evolution, and metabolic role of a novel glycolytic GAPDH enzyme recruited by land plant plastids. J. Mol. Evol. 57, 16-26 (2003).

38. Muñoz-Bertomeu, J. et al. The plastidial glyceraldehyde-3-phosphate dehydrogenase is critical for viable pollen development in Arabidopsis. Plant Physiol. 152, 1830-1841 (2010).

39. Rius, S. P., Casati, P., Iglesias, A. A. \& Gomez-Casati, D. F. Characterization of an Arabidopsis thaliana mutant lacking a cytosolic non-phosphorylating glyceraldehyde-3-phosphate dehydrogenase. Plant Mol. Biol. 61, 945-957 (2006).

40. Ralser, M. et al. Dynamic rerouting of the carbohydrate flux is key to counteracting oxidative stress. J. Biol. 6, 10 (2007).

41. Tristan, C., Shahani, N., Sedlak, T. W. \& Sawa, A. The diverse functions of GAPDH: views from different subcellular compartments. Cell. Signal. 23, 317-323 (2011).

42. Kim, S.-C., Nusinow, D. A., Sorkin, M. L., Pruneda-Paz, J. \& Wang, X. Interaction and regulation between lipid mediator phosphatidic acid and circadian clock regulators in Arabidopsis. Plant Cell 31, 399-416 (2019).

43. Clarke, L. \& Carbon, J. A colony bank containing synthetic CoI EI hybrid plasmids representative of the entire E. coli genome. Cell 9, 91-99 (1976).

44. Walter, M. et al. Visualization of protein interactions in living plant cells using bimolecular fluorescence complementation. Plant J. 40, 428-438 (2004).

45. Yoo, S. D., Cho, Y. H. \& Sheen, J. Arabidopsis mesophyll protoplasts: a versatile cell system for transient gene expression analysis. Nat. Protoc. 2, 1565-1572 (2007).

46. Lee, J. H., Jin, S., Kim, S. Y., Kim, W. \& Ahn, J. H. A fast, efficient chromatin immunoprecipitation method for studying protein-DNA binding in Arabidopsis mesophyll protoplasts. Plant Methods 13, 42 (2017).

47. Perez-Riverol, Y. et al. The PRIDE database and related tools and resources in 2019: improving support for quantification data. Nucleic Acids Res. 47, D442-D450 (2019).

\section{Acknowledgements}

We thank Dr. Nobutaka Mitsuda (National Institute of Advanced Industrial Science and Technology, Tsukuba, Japan) for his generous donation of the Arabidopsis transcription factor cDNA library and Dr. Sona Pandey (Donald Danforth Plant Science Center) for GPA1-YFP ${ }^{N}$ and PLDa1-YFPC constructs. This work was supported by grants from the National Science Foundation (MCB-1412901 to X.W.), the U.S. Department of Energy (DE-SC0001295 to X.W.) and the Agriculture and Food Research Initiative (AFRI) award no. (2016-67013-24429/project accession number 1007600 to X.W.) from the USDA National Institute of Food and Agriculture.

\section{Author contributions}

S.K. designed and performed all experiments, collected and analyzed data, and wrote the manuscript. L.G. generated GAPC-KO and OE mutants. X.W. proposed and supervised the study and edited the manuscript. All authors discussed the results and commented on the manuscript.

\section{Competing interests}

The authors declare no competing interests.

\section{Additional information}

Supplementary information is available for this paper at https://doi.org/10.1038/s41467 020-17311-4.

Correspondence and requests for materials should be addressed to X.W.

Peer review information Nature Communications thanks Diego Gomez-Casati, Renate Scheibe, Paolo Trost, and the other, anonymous reviewer(s) for their contribution to the peer review of this work.

Reprints and permission information is available at http://www.nature.com/reprints

Publisher's note Springer Nature remains neutral with regard to jurisdictional claims in published maps and institutional affiliations.

(c) (i) Open Access This article is licensed under a Creative Commons Attribution 4.0 International License, which permits use, sharing, adaptation, distribution and reproduction in any medium or format, as long as you give appropriate credit to the original author(s) and the source, provide a link to the Creative Commons license, and indicate if changes were made. The images or other third party material in this article are included in the article's Creative Commons license, unless indicated otherwise in a credit line to the material. If material is not included in the article's Creative Commons license and your intended use is not permitted by statutory regulation or exceeds the permitted use, you will need to obtain permission directly from the copyright holder. To view a copy of this license, visit http://creativecommons.org/ licenses/by/4.0/.

(C) The Author(s) 2020 